\title{
Finite element approximation of spectral acoustic problems on curved domains
}

\author{
Erwin Hernández ${ }^{1 \star}$ and Rodolfo Rodríguez ${ }^{2 \star \star}$ \\ 1 Departamento de Matemática, Universidad Técnica Federico Santa María, Casilla 110-V, \\ Valparaíso, Chile. E-mail: erwin.hernandez@mat.utfsm.cl \\ $2 \mathrm{GI}^{2} \mathrm{MA}$, Departamento de Ingeniería Matemática, Universidad de Concepción, Casilla \\ 160-C, Concepción, Chile. E-mail: rodolfo@ing-mat.udec.cl
}

\begin{abstract}
Summary. This paper deals with the finite element approximation of the displacement formulation of the spectral acoustic problem on a curved non convex two-dimensional domain $\Omega$. Convergence and error estimates are proved for Raviart-Thomas elements on a discrete polygonal domain $\Omega_{h} \not \subset$ $\Omega$ in the framework of the abstract spectral approximation theory. Similar results have been previously proved only for polygonal domains. Numerical tests confirming the theoretical results are reported.
\end{abstract}

Mathematics Subject Classification (1991): 65N25, 65N30, 70J30

Key words Acoustic vibrations, finite element spectral approximation, curved domains.

\section{Introduction}

A considerable amount of work has been devoted during the last years to devise and analyze efficient numerical tools to deal with structural-acoustic interaction problems. See for instance the monographs by Morand and Ohayon [29], Conca et al. [15], and Ohayon and Soize [30], where different mechanical problems involving fluid-structure interaction are treated. These references also include numerical techniques and extensive bibliographic lists.

Although the typical variable to describe an acoustic fluid is its pressure, since long time ago (see for instance [27]) practitioners recognize several

\footnotetext{
* Supported by FONDECYT 2000114 (Chile)

$\star \star$ Supported by FONDAP in Applied Mathematics (Chile).
} 
advantages of using fluid displacement formulations in spectral problems involving interaction with a solid. However, this approach has also a severe drawback: the presence of spurious vibration modes when standard finite elements are used for the fluid displacements.

Several alternatives have been proposed to avoid these spurious modes (see for instance [24, 13,38,21]). One of them was introduced in [8] and analyzed in $[3,31]$ for the structural-acoustic vibration problem. It is based on using Raviart-Thomas elements to discretize the fluid displacement fielda natural choice since the variational formulation is posed on $\mathrm{H}(\mathrm{div})$. Since then, this approach was successfully extended to deal with other spectral problems involving, for instance, incompressible fluids $[4,5]$, dissipative acoustics [6,9], interaction with slender structures [19,7], etc.

In all these cases, optimal order error estimates have been proved for eigenvalues and eigenfunctions. However, in all the proofs, the domain has been assumed to be polygonal (or polyhedral in 3D). The main reason for this assumption was to avoid some intrinsic difficulties in the application of the standard spectral approximation theory ([2]). These difficulties arise from the variational crime committed by approximating the curved domain with a polygonal one.

Even in much simpler cases like the finite element approximation of the spectral problem for the Laplacian on a general curved domain, the number of papers with theoretical results is remarkably small. Indeed, the first rigorous proofs for this problem valid for non-convex domains have been given just a few years ago by Vanmaele and Ženíšek ([35-37]) (using the min-max characterization; see [34,32]) and Lebaud ([28]) (using the abstract approximation theory; see [2]). These papers deal only with Dirichlet boundary conditions. For Neumann boundary conditions the first results have been obtained even more recently ([25]).

In the present paper we consider the vibration problem for an acoustic fluid contained within a rigid curved cavity. In spite of the fact that this problem can be efficiently solved by using pressure variables, we consider a displacement formulation of the fluid. The reason for this is the interest of applying this approach to fluid-structure vibration problems, where this formulation is convenient as remarked above. We prove spectral convergence and error estimates for eigenfunctions and eigenvalues, the latter of double-order as usual in this kind of problems.

From the mathematical point of view, the displacement formulation of the fluid acoustic vibration problem and its discretization with RaviartThomas elements is completely equivalent to the discretization with these elements of the mixed formulation of the spectral problem for the Laplacian (see $[1,18]$ ). Thus, our analysis also covers this more standard problem. Let us recall that although error estimates for this spectral problem on polygo- 
nal domains have been given in [2], some recent papers ([10,11]) show that, even in this case, it is not immediate to extend standard results on mixed methods to spectral problems.

The outline of the paper is as follows: in the following section we introduce the displacement formulation of the acoustic vibration problem and its discretization by Raviart-Thomas elements. In section 3, corresponding continuous and discrete operators are defined on a same Hilbert space, which allows us to fit this problem in the framework of the abstract spectral approximation theory ([2]). Let us remark that the correct definition of these operators is a key point for curved boundaries, because the continuous and discrete problems are posed on different domains. In section 4 we prove convergence in $\mathrm{H}$ (div)-norm of the discrete operators to the continuous one and apply this to obtain error estimates for the eigenfunctions. Since in this case an improved order of convergence in $\mathrm{L}^{2}$-norm cannot be expected, an additional approximation property has to be proved to obtain a double-order error estimate for the eigenvalues. Finally, in section 5, we report some numerical tests which confirm the theoretical results and exhibit the performance of the method.

Throughout the paper $C$ will denote a positive constant not necessarily the same at each occurrence but always independent of the mesh-size $h$.

\section{Statement of the problem}

We consider the problem of determining the vibrations modes of an ideal inviscid barotropic fluid contained in a rigid cavity, described by means of the fluid displacement field.

Let $\Omega \subset \mathbb{R}^{2}$ be the domain occupied by the fluid. We assume that $\Omega$ is a bounded open domain, in general non convex, with a Lipschitz boundary $\partial \Omega$. We denote by $\nu$ the outward unit normal to $\partial \Omega$. We also assume $\partial \Omega$ is piecewise smooth; more precisely, $\partial \Omega=\bigcup_{j=1}^{J} \Gamma_{j}$ with $\Gamma_{j}$ being $\mathcal{C}^{2}$ curves.

Consider the spectral problem which consists in finding $\lambda \in \mathbb{R}$ and $u \neq 0$ satisfying

$$
\left\{\begin{aligned}
-\nabla\left(\rho c^{2} \operatorname{div} u\right)=\lambda \rho u & \text { in } \Omega, \\
u \cdot \nu=0 & \text { on } \partial \Omega .
\end{aligned}\right.
$$

The solution of this problem are the free vibration modes of the fluid contained in $\Omega ; u$ is the amplitude of the displacement oscillation and $\sqrt{\lambda}$ the natural vibration frequency. On the other hand $\rho$ denotes the fluid density and $c$ the acoustic speed. Without loosing generality we assume $\rho=1$ and $c=1$.

Let $\mathrm{H}(\operatorname{div}, \Omega):=\left\{v \in \mathrm{L}^{2}(\Omega)^{2}: \operatorname{div} v \in \mathrm{L}^{2}(\Omega)\right\}$, which endowed with the norm defined by $\|v\|_{\operatorname{div}, \Omega}^{2}:=\|v\|_{0, \Omega}^{2}+\|\operatorname{div} v\|_{0, \Omega}^{2}$ is a Hilbert 
space. We denote

$$
\mathcal{V}:=\mathrm{H}_{0}(\operatorname{div}, \Omega):=\{v \in \mathrm{H}(\operatorname{div}, \Omega): v \cdot \nu=0 \text { on } \partial \Omega\},
$$

which is a closed subspace of $\mathrm{H}(\operatorname{div}, \Omega)$. Then, the variational formulation of the spectral problem above reads:

Find $\lambda \in \mathbb{R}$ and $u \in \mathcal{V}, u \neq 0$, such that

$$
\int_{\Omega} \operatorname{div} u \operatorname{div} v d x=\lambda \int_{\Omega} u \cdot v d x \quad \forall v \in \mathcal{V} .
$$

The above problem has exactly two types of solutions (see [3]):

1. $\lambda_{0}=0$, with corresponding eigenspace

$$
\mathcal{K}:=\left\{u \in \mathrm{H}_{0}(\operatorname{div}, \Omega): \operatorname{div} u=0 \text { in } \Omega\right\} ;
$$

2. a sequence of finite multiplicity eigenvalues $\lambda_{n}>0, n \in \mathbb{N}$, diverging to $+\infty$, with corresponding eigenfunctions $u_{n} \in \mathcal{V}$ satisfying $u_{n}=$ $\nabla \varphi_{n}$ for some $\varphi_{n} \in \mathrm{H}^{1}(\Omega)$.

The eigenfunctions of the second type form a complete orthogonal system of the closed subspace $\mathcal{G}$ of $\mathcal{V}$, which consists of the conservative displacement fields of this space; namely,

$$
\mathcal{G}:=\left\{u \in \mathrm{H}_{0}(\operatorname{div}, \Omega): u=\nabla \varphi, \varphi \in \mathrm{H}^{1}(\Omega)\right\} .
$$

Notice that $\mathcal{G}$ and $\mathcal{K}$ are orthogonal in $\mathrm{L}^{2}(\Omega)^{2}$ and $\mathrm{H}(\operatorname{div}, \Omega)$.

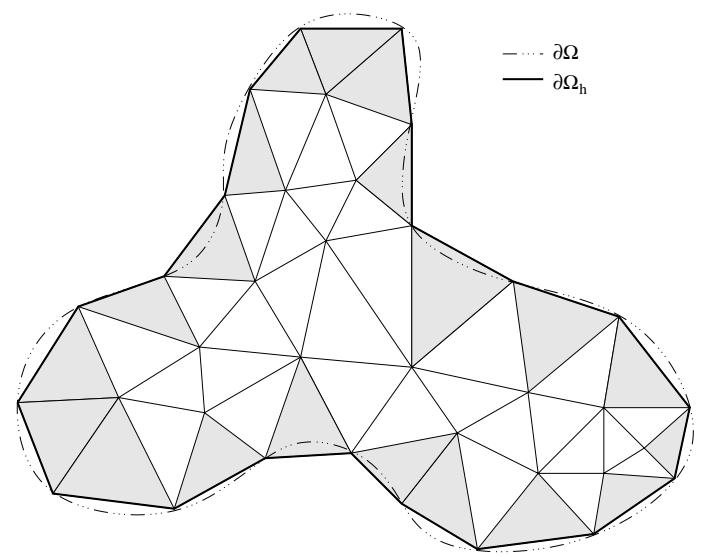

Fig. 1. Triangulation of $\Omega_{h} \approx \Omega$. The shaded triangles are the boundary ones $T \in \mathcal{T}_{h}^{\partial}$.

We consider a family $\left\{\mathcal{T}_{h}\right\}$ of standard finite element triangulations of polygonal domains $\Omega_{h}$ approximating $\Omega$ (see for instance [14]) such that, if $\mathcal{N}_{h}$ is the set of vertexes of all the triangles in $\mathcal{T}_{h}$, then there holds (see Fig. 1): 
- $\mathcal{N}_{h} \subset \bar{\Omega}_{h}$ and $\mathcal{N}_{h} \cap \partial \Omega_{h} \subset \partial \Omega$

- $\mathcal{N}_{h}$ contains all the points where the boundary $\partial \Omega$ is not $\mathcal{C}^{2}$;

- for all $T \in \mathcal{T}_{h}$, at most two vertexes of $T$ lie on $\partial \Omega_{h}$;

the third assumption is made only for the sake of simplicity.

As usual, $h$ stands for the mesh-size, namely, the maximum diameter $h_{T}$ of all the triangles $T \in \mathcal{T}_{h}$. We also assume that the family $\left\{\mathcal{T}_{h}\right\}$ is regular in the sense of a minimum angle condition; i.e., there exists $\theta_{0}>0$ such that, if $\theta_{T}$ is the smallest angle of $T$, then $\theta_{T}>\theta_{0}$ for all $T \in \mathcal{T}_{h}$ and for all the triangulations $\mathcal{T}_{h}$ in the family.

In what follows we will use some notation and definitions introduced in [20] (see also [39]). For a given triangulation $\mathcal{T}_{h}$, we denote by $\mathcal{T}_{h}^{\partial}$ the subset of the so called boundary triangles; namely, those having an edge on $\partial \Omega_{h}$ (see Fig. 1). For one such triangle $T$, let $P_{1}^{T}, P_{2}^{T}$, and $P_{3}^{T}$ be its three vertexes, with $s_{T}:=\overline{P_{2}^{T} P_{3}^{T}} \subset \partial \Omega_{h}$, and let $\sigma_{T}$ be the piece of $\partial \Omega$ approximated by $s_{T}$ (see Fig. 2).

$$
\text { Case: } \widetilde{T} \supset T
$$

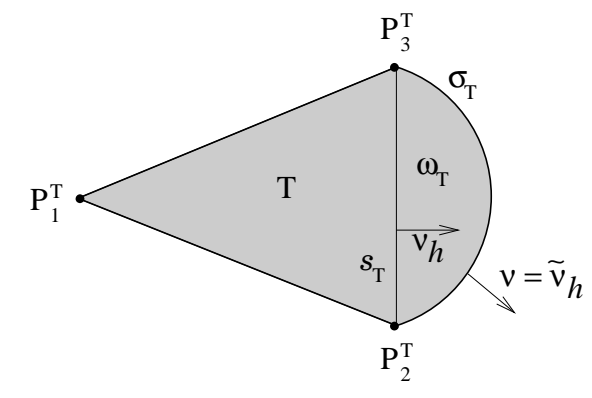

Fig. 2. Ideal triangles $\widetilde{T}$.
Case: $\widetilde{T} \subset T$

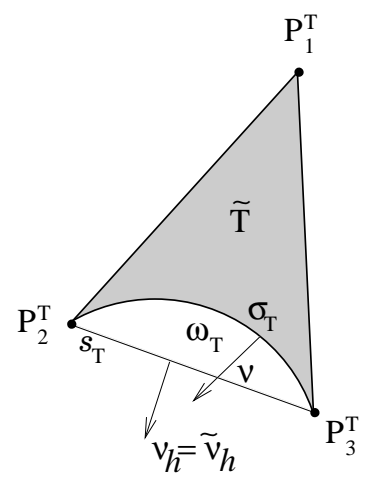

We denote by $\widetilde{T}$ the curved triangle of edges $\overline{P_{1}^{T} P_{2}^{T}}, \sigma_{T}$, and $\overline{P_{3}^{T} P_{1}^{T}}$, and we call it the ideal triangle associated with $T$. For the sake of simplicity we assume that the triangulations $\mathcal{T}_{h}$ are such that, for each boundary triangle $T \in \mathcal{T}_{h}^{\partial}$, either $T \subset \widetilde{T}$ or $T \supset \widetilde{T}$.

We consider the finite dimensional space $\mathcal{V}_{h}$ defined by

$$
\mathcal{V}_{h}:=\left\{v_{h} \in \mathcal{R}_{h}\left(\Omega_{h}\right):\left.v_{h} \cdot \nu_{h}\right|_{\partial \Omega_{h}}=0\right\},
$$


where $\nu_{h}$ is the outward unit normal to $\partial \Omega_{h}$ and $\mathcal{R}_{h}\left(\Omega_{h}\right)$ is the RaviartThomas space (see [33])

$$
\mathcal{R}_{h}\left(\Omega_{h}\right):=\left\{v_{h} \in \mathrm{H}\left(\operatorname{div}, \Omega_{h}\right):\left.v_{h}\right|_{T} \in \mathcal{R} \mathcal{T}_{0}(T) \forall T \in \mathcal{T}_{h}\right\},
$$

with

$$
\begin{aligned}
\mathcal{R} \mathcal{T}_{0}(T):=\left\{v_{h} \in \mathcal{P}_{1}(T)^{2}: v_{h}(x, y)=(a+b x, c+b y),\right. & \\
& a, b, c \in \mathbb{R},(x, y) \in T\} .
\end{aligned}
$$

Then, the discretization of the spectral problem (2.1) obtained by using these elements reads:

Find $\lambda_{h} \in \mathbb{R}$ and $u_{h} \in \mathcal{V}_{h}, u_{h} \neq 0$, such that

$$
\int_{\Omega_{h}} \operatorname{div} u_{h} \operatorname{div} v_{h} d x=\lambda_{h} \int_{\Omega_{h}} u_{h} \cdot v_{h} d x \quad \forall v_{h} \in \mathcal{V}_{h} .
$$

Proceeding as in [3], it is simple to prove that problem (2.2) has also two kinds of solutions:

1. $\lambda_{h 0}=0$, with corresponding eigenspace

$$
\mathcal{K}_{h}:=\left\{u_{h} \in \mathcal{V}_{h}: \operatorname{div} u_{h}=0 \text { in } \Omega_{h}\right\} ;
$$

2. a finite set of positive eigenvalues $\lambda_{h n}$, with corresponding eigenfunctions $u_{h n} \in \mathcal{G}_{h}$, where $\mathcal{G}_{h}$ is the orthogonal complement of $\mathcal{K}_{h}$ in $\mathcal{V}_{h}$.

Notice that $\mathcal{G}_{h}$ and $\mathcal{K}_{h}$ are also orthogonal in $\mathrm{L}^{2}\left(\Omega_{h}\right)^{2}$; i.e.,

$$
\mathcal{G}_{h}=\left\{u_{h} \in \mathcal{V}_{h}: \int_{\Omega_{h}} u_{h} \cdot v_{h} d x=0 \forall v_{h} \in \mathcal{K}_{h}\right\} .
$$

The goal of this paper is to prove that the solutions of the eigenvalue problem (2.2) approximate those of problem (2.1) and to establish error estimates.

\section{Linear operators associated with the spectral problems}

To study the convergence properties of problem (2.2), we will use the abstract spectral approximation theory stated in [2]. To do this we need to define, on a same fixed domain, operators $A$ and $A_{h}$ with spectra related to those of problems (2.1) and (2.2), respectively.

For each mesh $\mathcal{T}_{h}$ we denote

$$
\widetilde{\Omega}_{h}:=\Omega \cup \Omega_{h} .
$$

We also denote by $\widetilde{\nu}_{h}$ the outward unit normal to $\widetilde{\Omega}_{h}$. Notice that either $\widetilde{\nu}_{h}=\nu_{h}$ or $\widetilde{\nu}_{h}=\nu$ (see Fig. 2). 
We consider the operator $A$ defined by

$$
\begin{aligned}
A: \mathrm{L}^{2}\left(\mathbb{R}^{2}\right)^{2} & \longrightarrow \mathrm{H}\left(\operatorname{div}, \mathbb{R}^{2}\right) \hookrightarrow \mathrm{L}^{2}\left(\mathbb{R}^{2}\right)^{2} \\
f & \longmapsto \bar{u}:\left.\bar{u}\right|_{\Omega}=u,
\end{aligned}
$$

where $u \in \mathcal{G}$ is the solution of

$$
\int_{\Omega} \operatorname{div} u \operatorname{div} v d x=\int_{\Omega} f \cdot v d x \quad \forall v \in \mathcal{G}
$$

and, from now on, $\bar{v}$ denotes the extension of a function $v$ by zero from its original domain to $\mathbb{R}^{2}$. Notice that, for $u \in \mathrm{H}_{0}(\operatorname{div}, \Omega), \bar{u} \in \mathrm{H}\left(\operatorname{div}, \mathbb{R}^{2}\right)$ although $\bar{u} \notin \mathrm{H}^{1}\left(\mathbb{R}^{2}\right)$.

This problem is well posed because the bilinear form $\int_{\Omega} \operatorname{div} u \operatorname{div} v d x$ is $\mathcal{V}$-elliptic on $\mathcal{G}$. Indeed, given $v \in \mathcal{G}$, let $\varphi \in \mathrm{H}^{1}(\Omega)$ be such that $v=$ $\nabla \varphi$; then $\varphi$ is solution of the compatible Neumann problem

$$
\left\{\begin{aligned}
\Delta \varphi=\operatorname{div} v & \text { in } \Omega, \\
\frac{\partial \varphi}{\partial \nu}=0 & \text { on } \partial \Omega .
\end{aligned}\right.
$$

Hence, because of Lax-Milgram Lemma, there exist $C>0$ such that

$$
\|v\|_{0, \Omega}=\|\nabla \varphi\|_{0, \Omega} \leq C\|\operatorname{div} v\|_{0, \Omega} \quad \forall v \in \mathcal{G} .
$$

Consequently there exist $\alpha>0$ such that

$$
\int_{\Omega}(\operatorname{div} v)^{2} d x \geq \alpha\|v\|_{\operatorname{div}, \Omega}^{2} \quad \forall v \in \mathcal{G} .
$$

Therefore, by virtue of Lax-Milgram Lemma again, it is clear that $A$ is a well-defined bounded operator and, if $\bar{u}=A f$, then

$$
\|\bar{u}\|_{\operatorname{div}, \mathbb{R}^{2}} \leq C\|f\|_{0, \mathbb{R}^{2}}
$$

Furthermore, as a consequence of classical a priori estimates for (3.2) $\varphi$ attains additional regularity. Let us recall the definition of fractional Sobolev spaces (see for instance [22]). For $r \in(0,1)$, let

$$
\|v\|_{r, \Omega}:=\left[\|v\|_{0, \Omega}^{2}+\int_{\Omega} \int_{\Omega} \frac{|v(x)-v(y)|^{2}}{|x-y|^{2+2 r}} d x d y\right]^{1 / 2}
$$

and let $\mathrm{H}^{r}(\Omega):=\left\{v \in \mathrm{L}^{2}(\Omega):\|v\|_{r, \Omega}<\infty\right\}$. Then, according to [23], $\nabla \varphi \in \mathrm{H}^{r}(\Omega)^{2}$ and

$$
\|\nabla \varphi\|_{r, \Omega} \leq C\|\operatorname{div} v\|_{0, \Omega}
$$


with $r \in(1 / 2,1]$ (in fact, $r=1$ if $\Omega$ has no reentrant corner, and $r<\frac{\pi}{\theta}$, with $\theta$ being the largest interior angle of $\Omega$, otherwise). In particular, for $\bar{u}=A f$, we have that $u=\left.\bar{u}\right|_{\Omega} \in \mathrm{H}^{r}(\Omega)^{2}$ and

$$
\|u\|_{r, \Omega} \leq C\|\operatorname{div} u\|_{0, \Omega} \leq C\|f\|_{0, \mathbb{R}^{2}} .
$$

Regarding further regularity of $\operatorname{div} u$ we have the following lemma:

Lemma 3.1. Given $f \in \mathrm{L}^{2}\left(\mathbb{R}^{2}\right)^{2}$, let $u \in \mathcal{G}$ be the solution of problem (3.1). Then,

$$
\int_{\Omega}[\nabla(\operatorname{div} u)+f] \cdot \nabla \xi d x=0 \quad \forall \xi \in \mathrm{H}^{1}(\Omega) .
$$

Furthermore, $\operatorname{div} u \in \mathrm{H}^{1}(\Omega)$ and

$$
\|\operatorname{div} u\|_{1, \Omega} \leq C\|f\|_{0, \mathbb{R}^{2}}
$$

with $C$ a positive constant independent of $f$.

Proof. For $f \in \mathrm{L}^{2}(\Omega)^{2}$ the following decomposition holds (see [22]): $f=$ $\nabla \psi+\eta$, with $\psi \in \mathrm{H}^{1}(\Omega) / \mathbb{R}$ and $\eta \in \mathcal{K}$; furthermore, $\|\nabla \psi\|_{0, \Omega} \leq\|f\|_{0, \Omega}$. Then, because of (3.1) and the $\mathrm{L}^{2}(\Omega)$-orthogonality of $\mathcal{K}$ and $\mathcal{G}$, we have

$$
\int_{\Omega} \operatorname{div} u \operatorname{div} v d x=\int_{\Omega} f \cdot v d x=\int_{\Omega} \nabla \psi \cdot v d x \quad \forall v \in \mathcal{G},
$$

and consequently,

$$
-\int_{\Omega} \nabla(\operatorname{div} u) \cdot v d x=\int_{\Omega} \nabla \psi \cdot v d x \quad \forall v \in \mathcal{G} .
$$

The above equality is valid for $v \in \mathcal{K}$ too. Hence, since $\mathcal{C}_{0}^{\infty}(\Omega) \subset \mathcal{G} \oplus \mathcal{K}$, there holds

$$
\nabla(\operatorname{div} u+\psi)=0 \text { in } \Omega,
$$

which together with (3.5) yield (3.10). On the other hand,

$\int_{\Omega}[\nabla(\operatorname{div} u)+f] \cdot \nabla \xi d x=\int_{\Omega} \nabla(\operatorname{div} u+\psi) \cdot \nabla \xi d x+\int_{\Omega} \eta \cdot \nabla \xi d x=0$, and we conclude the proof.

It is simple to show that $\lambda_{n}$ is a positive eigenvalue of (2.1) if and only if $\mu_{n}=1 / \lambda_{n}$ is a positive eigenvalue of the operator $A$; moreover, the corresponding associated eigenfunctions are related by $u_{n}=\left.\bar{u}_{n}\right|_{\Omega}$. Since the eigenfunctions $u_{n}, n \in \mathbb{N}$, satisfy $-\nabla\left(\operatorname{div} u_{n}\right)=\lambda u_{n}$ in $\Omega$, according to (3.8), $u_{n} \in \mathrm{H}^{r}(\Omega)^{2}$ with $\left\|u_{n}\right\|_{r, \Omega} \leq C\left\|u_{n}\right\|_{0, \Omega}$. Consequently, from the previous lemma,

$$
\left\|\operatorname{div} u_{n}\right\|_{1+r, \Omega} \leq C\left\|u_{n}\right\|_{0, \Omega}
$$


with $r \in(1 / 2,1]$ being the constant in (3.8).

To define the discrete analogue of the operator $A$, we are going to prove that the bilinear form $\int_{\Omega_{h}} \operatorname{div} u_{h} \operatorname{div} v_{h} d x$ is also $\mathcal{V}$-elliptic on $\mathcal{G}_{h}$. To this goal, we first recall the following two lemmas stated in [25]:

Lemma 3.2. Given $T \in \mathcal{T}_{h}^{\partial}$, let $\omega_{T}$ be the domain bounded by $\sigma_{T}$ and $s_{T}$ (see Fig. 2). Then, there exists a constant $C>0$, independent of $T$, such that

$$
\left|\omega_{T}\right| \leq C h_{T}^{3}
$$

Consequently,

$$
\left|\Omega \backslash \bar{\Omega}_{h}\right|+\left|\Omega_{h} \backslash \bar{\Omega}\right| \leq C h^{2} .
$$

Proof. The first estimate is a direct consequence of the assumed smoothness of $\partial \Omega$ and standard interpolation results (see for instance [20]). Since $\left(\Omega \backslash \bar{\Omega}_{h}\right) \cup\left(\Omega_{h} \backslash \bar{\Omega}\right)=\bigcup\left\{\omega_{T}: T \in \mathcal{T}_{h}^{\partial}\right\}$, we obtain the second estimate by adding $\left|\omega_{T}\right|$ for all $T \in \mathcal{T}_{h}^{\partial}$.

In what follows we will have to prove several estimates of the type

$$
\|L v\| \leq C h^{s}\|v\|_{s, \Omega_{h}} \quad \forall v \in \mathrm{H}^{s}\left(\Omega_{h}\right),
$$

for different operators $L$ (v.g., (3.13), (3.16), and (3.17), below). To this aim, we will prove the above estimate for $s=0$ and $s=1$. Then, we will use the real method of interpolation of Sobolev spaces (see for instance Chapter 12 in [12]) to conclude

$$
\|L v\| \leq C h^{s}\|v\|_{\left[\mathrm{L}^{2}\left(\Omega_{h}\right), \mathrm{H}^{1}\left(\Omega_{h}\right)\right]_{s}},
$$

where $\|\cdot\|_{\left[\mathrm{L}^{2}\left(\Omega_{h}\right), \mathrm{H}^{1}\left(\Omega_{h}\right)\right]_{s}}$ is a norm in $\mathrm{H}^{s}\left(\Omega_{h}\right)$ which do not coincide with the intrinsic norm $\|\cdot\|_{s, \Omega_{h}}$ as defined by (3.6). However, according to Theorem 12.2.3 in [12],

$$
\|v\|_{\left[\mathrm{L}^{2}\left(\Omega_{h}\right), \mathrm{H}^{1}\left(\Omega_{h}\right)\right]_{s}} \leq C_{0}\left\|E_{G}\right\|\|v\|_{s, \Omega_{h}},
$$

where $C_{0}$ is a constant not depending on $\Omega_{h}$ and $E_{G}: \Omega_{h} \longrightarrow \mathbb{R}^{2}$ is the extension operator defined in [17]. Although $\left\|E_{G}\right\|$ depends on the domain $\Omega_{h}$, the arguments in this reference show that it is uniformly bounded with respect to $h$ as far as the angles of the polygonal domain $\Omega_{h}$ remain bounded away from $\pi$. This is our case since we have assumed that $\partial \Omega$ is piecewise $\mathcal{C}^{2}$.

Lemma 3.3. There exists a positive constant $C$ such that:

$$
\begin{array}{ccc}
\|v\|_{0, \Omega \backslash \bar{\Omega}_{h}} \leq C h^{s}\|v\|_{s, \Omega} & \forall v \in \mathrm{H}^{s}(\Omega) & (0 \leq s \leq 1) \\
\|v\|_{0, \Omega_{h} \backslash \bar{\Omega}} \leq C h^{s}\|v\|_{s, \Omega_{h}} & \forall v \in \mathrm{H}^{s}\left(\Omega_{h}\right) & (0 \leq s \leq 1)
\end{array}
$$


Proof. In the proof of Lemma 5.2-3 in [32] it is shown that

$$
\forall v \in \mathrm{H}^{1}(\Omega): \quad\|v\|_{0, \Omega \backslash \bar{\Omega}_{h}} \leq C\left(h\|v\|_{0, \partial \Omega}+h^{2}|v|_{1, \Omega \backslash \bar{\Omega}_{h}}\right) .
$$

The same arguments yield

$$
\forall v \in \mathrm{H}^{1}\left(\Omega_{h}\right): \quad\|v\|_{0, \Omega_{h} \backslash \bar{\Omega}} \leq C\left(h\|v\|_{0, \partial \Omega}+h^{2}|v|_{1, \Omega_{h} \backslash \bar{\Omega}}\right) .
$$

Then (3.12) and (3.13) follows for $s=1$ from this inequalities and the standard trace theorem in $\Omega$. Since the inequalities are clearly true for $s=$ 0 , they follow for $0<s<1$ from standard results on interpolation in Sobolev spaces (see, for instance, Theorem 1.4 in [22]).

We also recall the definition and some properties of the Raviart-Thomas interpolant (see for example [33]). For $s>1 / 2$, let

$$
R: \mathrm{H}^{s}\left(\Omega_{h}\right)^{2} \cap \mathrm{H}\left(\operatorname{div}, \Omega_{h}\right) \longrightarrow \mathcal{R}_{h}\left(\Omega_{h}\right),
$$

where for each $v \in \mathrm{H}^{s}\left(\Omega_{h}\right)^{2}, R v$ is the unique vector field in $\mathcal{R}_{h}\left(\Omega_{h}\right)$ satisfying

$$
\int_{\ell} R v \cdot \nu_{\ell} d s=\int_{\ell} v \cdot \nu_{\ell} d s
$$

for every edge $\ell$ of $\mathcal{T}$ ( $\nu_{\ell}$ being a unit normal to $\ell$ ). The operator $R$ satisfies for all $T \in \mathcal{T}_{h}$

$$
\int_{T} \operatorname{div}(v-R v) d x=0 \quad \forall v \in \mathrm{H}^{s}\left(\Omega_{h}\right)^{2} \cap \mathrm{H}\left(\operatorname{div}, \Omega_{h}\right) .
$$

Moreover, it is well known (see for example [33]) that for $v \in \mathrm{H}^{s}\left(\Omega_{h}\right)^{2}$ such that $\operatorname{div} v \in \mathrm{H}^{s}\left(\Omega_{h}\right)$

$$
\begin{aligned}
\|v-R v\|_{0, \Omega_{h}} & \leq C h^{s}\|v\|_{s, \Omega_{h},} \\
\|\operatorname{div}(v-R v)\|_{0, \Omega_{h}} & \leq C h^{s}\|\operatorname{div} v\|_{s, \Omega_{h}} .
\end{aligned}
$$

In what follows, we will use a Helmholtz decomposition of functions in $\mathcal{G}_{h}$ extended by zero outside $\Omega_{h}$. Consider the following spaces defined in $\widetilde{\Omega}_{h}$ :

$$
\begin{aligned}
& \mathcal{K}\left(\widetilde{\Omega}_{h}\right):=\left\{v \in \mathrm{H}_{0}\left(\operatorname{div}, \widetilde{\Omega}_{h}\right): \operatorname{div} v=0 \text { in } \widetilde{\Omega}_{h}\right\}, \\
& \mathcal{G}\left(\widetilde{\Omega}_{h}\right):=\left\{v \in \mathrm{H}_{0}\left(\operatorname{div}, \widetilde{\Omega}_{h}\right): v=\nabla \varphi, \varphi \in \mathrm{H}^{1}\left(\widetilde{\Omega}_{h}\right)\right\} .
\end{aligned}
$$

These two spaces are orthogonal in $\mathrm{H}\left(\operatorname{div}, \widetilde{\Omega}_{h}\right)$ and $\mathrm{L}^{2}\left(\widetilde{\Omega}_{h}\right)^{2}$. Notice that the inclusions $\mathcal{K} \hookrightarrow \mathcal{K}\left(\widetilde{\Omega}_{h}\right)$ and $\mathcal{K}_{h} \hookrightarrow \mathcal{K}\left(\widetilde{\Omega}_{h}\right)$ obtained by extending by zero the functions defined in $\Omega$ or $\Omega_{h}$, respectively, are continuous. 
In the following lemma, we are going to use the standard a priori estimate for a Neumann problem in $\widetilde{\Omega}_{h}$ :

$$
\left\{\begin{array}{cl}
\Delta \xi=g & \text { in } \widetilde{\Omega}_{h}, \\
\frac{\partial \xi}{\partial \widetilde{\nu}_{h}}=0 & \text { on } \partial \widetilde{\Omega}_{h} .
\end{array}\right.
$$

with $g \in \mathrm{L}^{2}\left(\widetilde{\Omega}_{h}\right)$ such that $\int_{\widetilde{\Omega}_{h}} g=0$. It is well known (see [23]) that $\nabla \xi \in$ $\mathrm{H}^{r_{h}}\left(\widetilde{\Omega}_{h}\right)$ with $r_{h} \in(1 / 2,1]$; in fact, $r_{h}=1$ if $\widetilde{\Omega}_{h}$ has no reentrant corner, and $r_{h}<\frac{\pi}{\theta_{h}}$, with $\theta_{h}$ being the largest interior angle of $\widetilde{\Omega}_{h}$, otherwise. Moreover,

$$
\|\nabla \xi\|_{r_{h}, \widetilde{\Omega}_{h}} \leq C\left(\widetilde{\Omega}_{h}\right)\|g\|_{0, \widetilde{\Omega}_{h}} .
$$

However, the available proofs provide a very poor control of the constant $C\left(\widetilde{\Omega}_{h}\right)$ (see Remark 4.3.2.6 in [23]). Because of this we need to make the following assumption:

Assumption 1 The domain $\Omega$ and the family of meshes $\left\{\mathcal{T}_{h}\right\}$ are such that the constants $C\left(\widetilde{\Omega}_{h}\right)$ in (3.18) are uniformly bounded with respect to $h$.

To the best of the authors' knowledge, this assumption has not been proved yet neither for a domain $\Omega$ with a $\mathcal{C}^{\infty}$ boundary. However, according to Costabel et al. [16], this is a plausible hypothesis for any Lipschitz domain $\Omega$ with a piecewise $\mathcal{C}^{2}$ boundary. Indeed, their conjecture is that, for such domain, it could be proved via a boundary integral formulation that the constants $C\left(\widetilde{\Omega}_{h}\right)$ are uniformly bounded with respect to $h$.

Lemma 3.4. For any $v_{h} \in \mathcal{G}_{h}$,

$$
\bar{v}_{h}=\nabla \xi+\chi \quad \text { in } \widetilde{\Omega}_{h},
$$

with $\nabla \xi \in \mathcal{G}\left(\widetilde{\Omega}_{h}\right)$ and $\chi \in \mathcal{K}\left(\widetilde{\Omega}_{h}\right)$. Moreover, there exist constants $C>0$ and $r_{h} \in(1 / 2,1]$, both independent of $v_{h}$, such that $\nabla \xi \in \mathrm{H}^{r_{h}}\left(\widetilde{\Omega}_{h}\right)$,

$$
\|\nabla \xi\|_{r_{h}, \widetilde{\Omega}_{h}} \leq C\left\|\operatorname{div} v_{h}\right\|_{0, \Omega_{h}},
$$

and

$$
\|\chi\|_{0, \widetilde{\Omega}_{h}} \leq C h^{r_{h}}\left\|\operatorname{div} v_{h}\right\|_{0, \Omega_{h}} .
$$

Proof. Let $\xi \in \mathrm{H}^{1}\left(\widetilde{\Omega}_{h}\right) / \mathbb{R}$ be the solution of the compatible Neumann problem

$$
\left\{\begin{aligned}
\Delta \xi=\operatorname{div} \bar{v}_{h} & \text { in } \widetilde{\Omega}_{h}, \\
\frac{\partial \xi}{\partial \widetilde{\nu}_{h}}=0 & \text { on } \partial \widetilde{\Omega}_{h} .
\end{aligned}\right.
$$

Then, (3.19) holds true because of Assumption 1. 
Let $\chi:=\bar{v}_{h}-\nabla \xi$ in $\widetilde{\Omega}_{h}$. Then $\chi \in \mathcal{K}\left(\widetilde{\Omega}_{h}\right)$, since $\operatorname{div} \chi=0$ in $\widetilde{\Omega}_{h}$ and $\chi \cdot \widetilde{\nu}_{h}=\bar{v}_{h} \cdot \widetilde{\nu}_{h}-\nabla \xi \cdot \widetilde{\nu}_{h}=0$ on $\partial \widetilde{\Omega}_{h}$.

On the other hand, since $\left.\bar{v}_{h}\right|_{\Omega \backslash \bar{\Omega}_{h}}=0$, we have

$$
\begin{aligned}
\int_{\widetilde{\Omega}_{h}}|\chi|^{2} d x= & \int_{\Omega_{h}} \chi \cdot\left(v_{h}-\nabla \xi\right) d x-\int_{\Omega \backslash \bar{\Omega}_{h}} \chi \cdot \nabla \xi d x \\
= & \int_{\Omega_{h}} \chi \cdot\left(v_{h}-R \nabla \xi\right) d x+\int_{\Omega_{h}} \chi \cdot(R \nabla \xi-\nabla \xi) d x \\
& -\int_{\Omega \backslash \bar{\Omega}_{h}} \chi \cdot \nabla \xi d x .
\end{aligned}
$$

Thus, to prove the lemma, we estimate the three terms in the right-hand side of this equality.

For the first one, we note that for $T \in \mathcal{T}_{h}^{\partial}$ such that $T \subset \widetilde{T}$ (see Fig. 2), we have

$$
\int_{\sigma_{T}} \frac{\partial \xi}{\partial \nu} d s+\int_{s_{T}} \frac{\partial \xi}{\partial \nu_{h}} d s=\int_{\omega_{T}} \Delta \xi d x=\int_{\widetilde{T} \backslash T} \operatorname{div} \bar{v}_{h} d x=0 .
$$

Hence,

$$
\int_{s_{T}} \frac{\partial \xi}{\partial \nu_{h}} d s=-\int_{\sigma_{T}} \frac{\partial \xi}{\partial \nu} d s=-\int_{\sigma_{T}} \frac{\partial \xi}{\partial \widetilde{\nu}_{h}} d s=0,
$$

because $\partial \xi / \partial \widetilde{\nu}_{h}$ vanishes on the whole $\partial \widetilde{\Omega}_{h} \supset \sigma_{T}$. On the other hand, for $T \in \mathcal{T}_{h}^{\partial}$ such that $T \supset \widetilde{T}$, we directly have $\partial \xi / \partial \nu_{h}=\partial \xi / \partial \widetilde{\nu}_{h}=0$ on $s_{T} \subset \partial \Omega_{h}$. Then, by the definition of $R,(R \nabla \xi) \cdot \nu_{h}$ vanishes on $s_{T}$ for all $T \in \mathcal{T}_{h}^{\partial}$. Hence $\left(v_{h}-R \nabla \xi\right) \cdot \nu_{h}=0$ on $\partial \Omega_{h}$. In addition we have $\left.\operatorname{div} v_{h}\right|_{T} \in \mathcal{P}_{0}(T)$ and $\left.\operatorname{div} \nabla \xi\right|_{T}=\left.\operatorname{div} v_{h}\right|_{T}$. Then it follows that $\left.\operatorname{div} \nabla \xi\right|_{T} \in \mathcal{P}_{0}(T)$ and, from (3.15),

$$
\left.\operatorname{div}(R \nabla \xi)\right|_{T}=\left.\operatorname{div} \nabla \xi\right|_{T}=\left.\operatorname{div} v_{h}\right|_{T} .
$$

Hence $\left(v_{h}-R \nabla \xi\right) \in \mathcal{K}_{h} \hookrightarrow \mathcal{K}\left(\widetilde{\Omega}_{h}\right)$ and then, since $v_{h} \in \mathcal{G}_{h}$ and $\nabla \xi \in$ $\mathcal{G}\left(\widetilde{\Omega}_{h}\right)$, we have

$$
\begin{aligned}
\int_{\Omega_{h}} \chi \cdot\left(v_{h}-R \nabla \xi\right) d x= & \int_{\Omega_{h}} v_{h} \cdot\left(v_{h}-R \nabla \xi\right) d x \\
& -\int_{\widetilde{\Omega}_{h}} \nabla \xi \cdot \overline{\left(v_{h}-R \nabla \xi\right)} d x=0 .
\end{aligned}
$$

For the second term, we use (3.16) and (3.19) to obtain

$$
\begin{aligned}
\left|\int_{\Omega_{h}} \chi \cdot(R \nabla \xi-\nabla \xi) d x\right| & \leq\|\chi\|_{0, \Omega_{h}} C h^{r_{h}}\|\nabla \xi\|_{r_{h}, \Omega_{h}} \\
& \leq C h^{r_{h}}\|\chi\|_{0, \widetilde{\Omega}_{h}}\left\|\operatorname{div} v_{h}\right\|_{0, \Omega_{h}} .
\end{aligned}
$$


Finally, the last term is bounded by using Lemma 3.3 and (3.19) as follows,

$$
\begin{aligned}
\left|\int_{\Omega \backslash \bar{\Omega}_{h}} \chi \cdot \nabla \xi d x\right| & \leq\|\chi\|_{0, \Omega \backslash \bar{\Omega}_{h}} C h^{r_{h}}\|\nabla \xi\|_{r_{h}, \widetilde{\Omega}_{h}} \\
& \leq C h^{r_{h}}\|\chi\|_{0, \widetilde{\Omega}_{h}}\left\|\operatorname{div} v_{h}\right\|_{0, \Omega_{h}} .
\end{aligned}
$$

As an immediate consequence of the previous lemma, we have the following result:

Corollary 3.1. There exists a positive constant $\alpha$, independent of $h$, such that

$$
\int_{\Omega_{h}}\left(\operatorname{div} v_{h}\right)^{2} d x \geq \alpha\left\|v_{h}\right\|_{\operatorname{div}, \Omega_{h}}^{2} \quad \forall v_{h} \in \mathcal{G}_{h} .
$$

Proof. Let $v_{h} \in \mathcal{G}_{h}$. According to the previous lemma, we have $\bar{v}_{h}=$ $\nabla \xi+\chi$ and

$$
\left\|v_{h}\right\|_{0, \Omega_{h}}=\left\|\bar{v}_{h}\right\|_{0, \widetilde{\Omega}_{h}} \leq\|\nabla \xi\|_{0, \widetilde{\Omega}_{h}}+\|\chi\|_{0, \widetilde{\Omega}_{h}} \leq C\left(1+h^{r_{h}}\right)\left\|\operatorname{div} v_{h}\right\|_{0, \Omega_{h}},
$$

which allows us to conclude the proof.

Now, we define the discrete analogue of the operator $A$ as follows:

$$
\begin{aligned}
A_{h}: \mathrm{L}^{2}\left(\mathbb{R}^{2}\right)^{2} & \longrightarrow \mathrm{L}^{2}\left(\mathbb{R}^{2}\right)^{2} \\
f & \longmapsto \bar{u}_{h}:\left.\bar{u}_{h}\right|_{\Omega_{h}}=u_{h},
\end{aligned}
$$

where $u_{h} \in \mathcal{G}_{h}$ is the solution of

$$
\int_{\Omega_{h}} \operatorname{div} u_{h} \operatorname{div} v_{h} d x=\int_{\Omega_{h}} f \cdot v_{h} d x \quad \forall v_{h} \in \mathcal{G}_{h}
$$

(recall that $\bar{u}_{h}$ denotes the extension by zero of $u_{h}$ to $\mathbb{R}^{2}$ ).

Problem (3.22) is well posed because of Corollary 3.1. Then, as a consequence of Lax-Milgram Lemma, $A_{h}$ is well defined and we have

$$
\left\|u_{h}\right\|_{\text {div }, \Omega_{h}} \leq C\|f\|_{0, \mathbb{R}^{2}} .
$$

As in the continuous case, it is simple to show that $\lambda_{h n}$ is a positive eigenvalue of problem (2.2) if and only if $\mu_{h n}=1 / \lambda_{h n}$ is a positive eigenvalue of the operator $A_{h}$, and the corresponding associated eigenfunctions are related by $u_{h n}=\left.\bar{u}_{h n}\right|_{\Omega}$. In the following section we study how the eigenvalues and eigenfunctions of $A_{h}$ approximate those of $A$. 
Remark 3.1. Under the assumption that $\partial \Omega$ is piecewise smooth, the constant $r_{h}$ in Lemma 3.4 satisfies $r_{h} \rightarrow r$ as $h$ goes to zero, with $r \in(1 / 2,1]$ being the constant of estimates (3.8) and (3.11). Indeed, if $\Omega$ is convex so is $\Omega_{h}$, and in this case clearly $r=r_{h}=1$. Otherwise, $r=\frac{\pi}{\theta}$ and $r_{h}=\frac{\pi}{\theta_{h}}$, with $\theta$ and $\theta_{h}$ being the largest reentrant corners of $\Omega$ and $\Omega_{h}$, respectively. Since $\Omega$ is assumed to be piecewise $\mathcal{C}^{2}$, then $\theta_{h} \rightarrow \theta$ as $h$ goes to zero. Therefore, if we denote $r_{h}^{\prime}:=\min \left\{r, r_{h}\right\}$, then $r_{h}^{\prime}$ is asymptotically equal to $r$. Because of this, we will use $r_{h}^{\prime}$ instead of $r$ or $r_{h}$ several times in the following section, just to simplify the notation.

\section{Spectral approximation}

Our first step is to prove that the operators $A_{h}$ converge to $A$ in norm as $h$ goes to zero.

From now on and throughout the rest of the paper, let $f \in \mathrm{L}^{2}\left(\mathbb{R}^{2}\right)^{2}$ be a fixed function and let

$$
\bar{u}=A f, \quad u=\left.\bar{u}\right|_{\Omega}, \quad \bar{u}_{h}=A_{h} f, \quad u_{h}=\left.\bar{u}_{h}\right|_{\Omega_{h}} .
$$

Furthermore, $C$ will denote a generic constant independent of the mesh-size as above, but also independent on the particular $f \in \mathrm{L}^{2}\left(\mathbb{R}^{2}\right)^{2}$.

We will use in the proofs smooth extensions of functions originally defined in $\Omega$. For $\psi \in \mathrm{H}^{s}(\Omega)(s>0)$, let $\psi^{e}$ denote an extension of $\psi$ to $\mathbb{R}^{2}$ satisfying $\psi^{e} \in \mathrm{H}^{s}\left(\mathbb{R}^{2}\right)$ and

$$
\left\|\psi^{e}\right\|_{s, \mathbb{R}^{2}} \leq C\|\psi\|_{s, \Omega}
$$

(see, for instance, Theorem 1.4.3.1 in [23]). In particular, since according to estimate (3.10) $\operatorname{div} u \in \mathrm{H}^{1}(\Omega),(\operatorname{div} u)^{e}$ denotes an extension of $\operatorname{div} u$ satisfying the estimate above for $s=1$; namely,

$$
\left\|(\operatorname{div} u)^{e}\right\|_{1, \mathbb{R}^{2}} \leq C\|\operatorname{div} u\|_{1, \Omega} .
$$

In addition, we introduce a gradient field defined in $\widetilde{\Omega}_{h}$, closely related to $u$ (as will be shown in Lemma 4.1 below). Let $\hat{\varphi} \in \mathrm{H}^{1}\left(\widetilde{\Omega}_{h}\right)$ be the solution of the following Neumann problem:

$$
\left\{\begin{aligned}
\Delta \hat{\varphi}=(\operatorname{div} u)^{e}+\hat{c} & \text { in } \widetilde{\Omega}_{h}, \\
\frac{\partial \hat{\varphi}}{\partial n}=0 & \text { on } \partial \widetilde{\Omega}_{h}, \\
\int_{\widetilde{\Omega}_{h}}^{\hat{\varphi}} \hat{\varphi}=0, &
\end{aligned}\right.
$$

where the constant $\hat{c}$ is taken for the problem to be compatible, i.e.,

$$
\hat{c}:=-\frac{1}{\left|\widetilde{\Omega}_{h}\right|} \int_{\widetilde{\Omega}_{h}}(\operatorname{div} u)^{e} d x=-\frac{1}{\left|\widetilde{\Omega}_{h}\right|} \int_{\Omega_{h} \backslash \bar{\Omega}}(\operatorname{div} u)^{e} d x
$$


Then, using Lemmas 3.2 and 3.3, and estimates (4.2) and (3.10), we have $|\hat{c}| \leq \frac{\left|\Omega_{h} \backslash \bar{\Omega}\right|^{1 / 2}}{\left|\widetilde{\Omega}_{h}\right|}\left\|(\operatorname{div} u)^{e}\right\|_{0, \Omega_{h} \backslash \bar{\Omega}} \leq C h^{2}\left\|(\operatorname{div} u)^{e}\right\|_{1, \Omega_{h}} \leq C h^{2}\|f\|_{0, \mathbb{R}^{2}}$.

Because of the a priori estimate for the Neumann problem (see [23]), $\hat{\varphi} \in$ $\mathrm{H}^{1+r_{h}}\left(\widetilde{\Omega}_{h}\right)$ and

$$
\|\hat{\varphi}\|_{1+r_{h}, \widetilde{\Omega}_{h}} \leq C\left\|(\operatorname{div} u)^{e}+\hat{c}\right\|_{0, \widetilde{\Omega}_{h}} \leq C\|f\|_{0, \mathbb{R}^{2}},
$$

the latter because of (4.2), (3.10), and (4.5). Thus, we define $\hat{u}:=\nabla \hat{\varphi}$. Then $\hat{u} \in \mathrm{H}^{r_{h}}\left(\widetilde{\Omega}_{h}\right)$ and

$$
\|\hat{u}\|_{r_{h}, \widetilde{\Omega}_{h}} \leq C\|f\|_{0, \mathbb{R}^{2}} .
$$

On the other hand, $\operatorname{div} \hat{u}=(\operatorname{div} u)^{e}+\hat{c} \in \mathrm{H}^{1}\left(\widetilde{\Omega}_{h}\right)$ and, because of (4.2), (3.10), and (4.5) again,

$$
\|\operatorname{div} \hat{u}\|_{1, \widetilde{\Omega}_{h}} \leq C\|f\|_{0, \mathbb{R}^{2}} .
$$

The following lemma shows that $\hat{u}$ is indeed an accurate approximation of $u$ in $\Omega \cap \Omega_{h}$ :

\section{Lemma 4.1. There exists a positive constant $C$ such that}

$$
\|u-\hat{u}\|_{\operatorname{div}, \Omega \cap \Omega_{h}} \leq C h^{\left(r+r_{h}^{\prime}\right) / 2}\|f\|_{0, \mathbb{R}^{2}} .
$$

Proof. Let $\varphi \in \mathrm{H}^{1}(\Omega)$ be the solution of the Neumann problem (3.2) for $v=u$, which satisfies $\int_{\Omega} \varphi=0$. Then, according to (3.7) and (3.5), $\varphi \in$ $\mathrm{H}^{1+r}(\Omega)$ and $\|\varphi\|_{1+r, \Omega} \leq C\|\operatorname{div} u\|_{0, \Omega} \leq C\|f\|_{0, \mathbb{R}^{2}}$.

Let $\varphi^{e}$ denote an extension to $\mathbb{R}^{2}$ of $\varphi \in \mathrm{H}^{1+r}(\Omega)$ satisfying (4.1) with $s=1+r$. Then

$$
\left\|\varphi^{e}\right\|_{1+r, \mathbb{R}^{2}} \leq C\|\varphi\|_{1+r, \Omega} \leq C\|f\|_{0, \mathbb{R}^{2}} .
$$

Recalling the weak formulation of problems (3.2) and (4.3), we have

$$
\begin{aligned}
\|u-\hat{u}\|_{0, \Omega \cap \Omega_{h}}^{2}= & \int_{\Omega \cap \Omega_{h}} \nabla(\varphi-\hat{\varphi}) \cdot \nabla(\varphi-\hat{\varphi}) d x \\
\leq & \int_{\widetilde{\Omega}_{h}} \nabla\left(\varphi^{e}-\hat{\varphi}\right) \cdot \nabla\left(\varphi^{e}-\hat{\varphi}\right) d x \\
\leq & \int_{\Omega} \nabla \varphi \cdot \nabla(\varphi-\hat{\varphi}) d x+\int_{\Omega_{h} \backslash \bar{\Omega}} \nabla \varphi^{e} \cdot \nabla\left(\varphi^{e}-\hat{\varphi}\right) d x \\
& -\int_{\widetilde{\Omega}_{h}} \nabla \hat{\varphi} \cdot \nabla\left(\varphi^{e}-\hat{\varphi}\right) d x
\end{aligned}
$$




$$
\begin{aligned}
\leq & -\int_{\Omega} \operatorname{div} u(\varphi-\hat{\varphi}) d x+\int_{\widetilde{\Omega}_{h}}(\operatorname{div} u)^{e}\left(\varphi^{e}-\hat{\varphi}\right) d x \\
& +\int_{\widetilde{\Omega}_{h}} \hat{c}\left(\varphi^{e}-\hat{\varphi}\right) d x+\int_{\Omega_{h} \backslash \bar{\Omega}} \nabla \varphi^{e} \cdot \nabla\left(\varphi^{e}-\hat{\varphi}\right) d x \\
= & \int_{\Omega_{h} \backslash \bar{\Omega}}(\operatorname{div} u)^{e}\left(\varphi^{e}-\hat{\varphi}\right) d x+\int_{\widetilde{\Omega}_{h}} \hat{c}\left(\varphi^{e}-\hat{\varphi}\right) d x \\
& +\int_{\Omega_{h} \backslash \bar{\Omega}} \nabla \varphi^{e} \cdot \nabla\left(\varphi^{e}-\hat{\varphi}\right) d x .
\end{aligned}
$$

Now, we estimate the three terms in the previous inequality. For the first one, we use Cauchy-Schwarz inequality, Lemma 3.3, (4.2), (3.10), (4.9), and (4.6) to obtain

$$
\begin{aligned}
\left|\int_{\Omega_{h} \backslash \bar{\Omega}}(\operatorname{div} u)^{e}\left(\varphi^{e}-\hat{\varphi}\right) d x\right| & \leq C h\left\|(\operatorname{div} u)^{e}\right\|_{1, \widetilde{\Omega}_{h}} C h\left\|\varphi^{e}-\hat{\varphi}\right\|_{1, \widetilde{\Omega}_{h}} \\
& \leq C h^{2}\|\operatorname{div} u\|_{1, \Omega}\left(\left\|\varphi^{e}\right\|_{1, \Omega}+\|\hat{\varphi}\|_{1, \widetilde{\Omega}_{h}}\right) \\
& \leq C h^{2}\|f\|_{0, \mathbb{R}^{2}}^{2} .
\end{aligned}
$$

For the second one, we use (4.5), (4.9), and (4.6), to obtain

$$
\left|\int_{\widetilde{\Omega}_{h}} \hat{c}\left(\varphi^{e}-\hat{\varphi}\right) d x\right| \leq C h^{2}\|f\|_{0, \mathbb{R}^{2}}\left(\|\varphi\|_{1, \Omega}+\|\hat{\varphi}\|_{1, \widetilde{\Omega}_{h}}\right) \leq C h^{2}\|f\|_{0, \mathbb{R}^{2}}^{2} \text {. }
$$

For the last term we use Cauchy-Schwarz inequality, Lemma 3.3, (4.9) and (4.6) to obtain

$$
\begin{aligned}
\mid \int_{\Omega_{h} \backslash \bar{\Omega}} \nabla \varphi^{e} \cdot \nabla\left(\varphi^{e}\right. & -\hat{\varphi}) d x \mid \\
& \leq C h^{r}\left\|\nabla \varphi^{e}\right\|_{r, \widetilde{\Omega}_{h}} C h^{r_{h}^{\prime}}\left\|\nabla\left(\varphi^{e}-\hat{\varphi}\right)\right\|_{r_{h}^{\prime}, \widetilde{\Omega}_{h}} \\
& \leq C h^{r+r_{h}^{\prime}}\|f\|_{0, \mathbb{R}^{2}}\left(\|\varphi\|_{1+r, \Omega}+\|\hat{\varphi}\|_{1+r_{h}, \mathbb{R}^{2}}\right) \\
& \leq C h^{r+r_{h}^{\prime}}\|f\|_{0, \mathbb{R}^{2}}^{2} .
\end{aligned}
$$

Finally, we note that $\left.\operatorname{div}(u-\hat{u})\right|_{\Omega \cap \Omega_{h}}=\hat{c}$. Then, using (4.5) and combining the above three inequalities, we have

$$
\|u-\hat{u}\|_{\operatorname{div}, \Omega \cap \Omega_{h}}^{2}=\|u-\hat{u}\|_{0, \Omega \cap \Omega_{h}}^{2}+\hat{c}^{2}\left|\Omega \cap \Omega_{h}\right| \leq C h^{r+r_{h}^{\prime}}\|f\|_{0, \mathbb{R}^{2}}^{2},
$$

which allows us to conclude the proof.

We have now the tools to prove the convergence in norm of $A_{h}$ to $A$. As a first step, the following lemma splits a bound of $\left\|\left(A-A_{h}\right) f\right\|_{\operatorname{div}, \mathbb{R}^{2}}=$ $\left\|\bar{u}-\bar{u}_{h}\right\|_{\text {div, } \mathbb{R}^{2}}$ into several terms which can be dealt with separately: 


\section{Lemma 4.2. There exists a positive constant $C$ such that}

$$
\begin{aligned}
\left\|\bar{u}-\bar{u}_{h}\right\|_{\operatorname{div}, \mathbb{R}^{2}} \leq C[ & \|u-\hat{u}\|_{\operatorname{div}, \Omega \cap \Omega_{h}}+\inf _{v_{h} \in \mathcal{G}_{h}}\left\|\hat{u}-v_{h}\right\|_{\operatorname{div}, \Omega_{h}} \\
& +\sup _{w_{h} \in \mathcal{G}_{h}} \frac{\left|\int_{\Omega_{h}} \operatorname{div}\left(\hat{u}-u_{h}\right) \operatorname{div} w_{h} d x\right|}{\left\|w_{h}\right\|_{\operatorname{div}, \Omega_{h}}} \\
& \left.+\|u\|_{\operatorname{div}, \Omega \backslash \bar{\Omega}_{h}}+\|\hat{u}\|_{\operatorname{div}, \Omega_{h} \backslash \bar{\Omega}}\right] .
\end{aligned}
$$

Proof. For all $v_{h} \in \mathcal{G}_{h}$

$$
\begin{aligned}
\left\|\bar{u}-\bar{u}_{h}\right\|_{\operatorname{div}, \mathbb{R}^{2}}^{2}= & \left\|u-u_{h}\right\|_{\operatorname{div}, \Omega \cap \Omega_{h}}^{2}+\|u\|_{\operatorname{div}, \Omega \backslash \bar{\Omega}_{h}}^{2}+\left\|u_{h}\right\|_{\operatorname{div}, \Omega_{h} \backslash \bar{\Omega}}^{2} \\
\leq & 2\left(\left\|u-v_{h}\right\|_{\operatorname{div}, \Omega \cap \Omega_{h}}^{2}+\left\|v_{h}-u_{h}\right\|_{\operatorname{div}, \Omega \cap \Omega_{h}}^{2}\right) \\
& +\|u\|_{\operatorname{div}, \Omega \backslash \bar{\Omega}_{h}}^{2}+\left\|u_{h}\right\|_{\operatorname{div}, \Omega_{h} \backslash \bar{\Omega}}^{2} .
\end{aligned}
$$

Then, using that $\left\|u_{h}\right\|_{\operatorname{div}, \Omega_{h} \backslash \bar{\Omega}} \leq\left\|v_{h}-u_{h}\right\|_{\operatorname{div}, \Omega_{h} \backslash \bar{\Omega}}+\left\|v_{h}\right\|_{\operatorname{div}, \Omega_{h} \backslash \bar{\Omega}}$, we have

$$
\begin{aligned}
\left\|\bar{u}-\bar{u}_{h}\right\|_{\operatorname{div}, \mathbb{R}^{2}} \leq C & \left(\left\|u-v_{h}\right\|_{\operatorname{div}, \Omega \cap \Omega_{h}}+\left\|v_{h}-u_{h}\right\|_{\operatorname{div}, \Omega_{h}}\right. \\
& \left.+\|u\|_{\operatorname{div}, \Omega \backslash \bar{\Omega}_{h}}+\left\|v_{h}\right\|_{\operatorname{div}, \Omega_{h} \backslash \bar{\Omega}}\right) .
\end{aligned}
$$

Because of the ellipticity estimate (3.21), we have

$$
\begin{aligned}
\alpha\left\|v_{h}-u_{h}\right\|_{\operatorname{div}, \Omega_{h}}^{2} \leq & \int_{\Omega_{h}} \operatorname{div}\left(v_{h}-\hat{u}\right) \operatorname{div}\left(v_{h}-u_{h}\right) d x \\
& +\int_{\Omega_{h}} \operatorname{div}\left(\hat{u}-u_{h}\right) \operatorname{div}\left(v_{h}-u_{h}\right) d x \\
\leq & \left\|v_{h}-\hat{u}\right\|_{\operatorname{div}, \Omega_{h}}\left\|v_{h}-u_{h}\right\|_{\operatorname{div}, \Omega_{h}} \\
& +\int_{\Omega_{h}} \operatorname{div}\left(\hat{u}-u_{h}\right) \operatorname{div}\left(v_{h}-u_{h}\right) d x
\end{aligned}
$$

then

$$
\begin{aligned}
\left\|v_{h}-u_{h}\right\|_{\operatorname{div}, \Omega_{h}} \leq \frac{1}{\alpha} & \left(\left\|v_{h}-\hat{u}\right\|_{\operatorname{div}, \Omega_{h}}\right. \\
& \left.\quad+\sup _{w_{h} \in \mathcal{G}_{h}} \frac{\left|\int_{\Omega_{h}} \operatorname{div}\left(\hat{u}-u_{h}\right) \operatorname{div} w_{h} d x\right|}{\left\|w_{h}\right\|_{\operatorname{div}, \Omega_{h}}}\right) .
\end{aligned}
$$


We conclude the proof by combining the above inequalities and the relations

$$
\left\|u-v_{h}\right\|_{\operatorname{div}, \Omega \cap \Omega_{h}} \leq\|u-\hat{u}\|_{\operatorname{div}, \Omega \cap \Omega_{h}}+\left\|\hat{u}-v_{h}\right\|_{\operatorname{div}, \Omega \cap \Omega_{h}}
$$

and

$$
\left\|v_{h}\right\|_{\operatorname{div}, \Omega_{h} \backslash \bar{\Omega}} \leq\left\|\hat{u}-v_{h}\right\|_{\operatorname{div}, \Omega_{h}}+\|\hat{u}\|_{\operatorname{div}, \Omega_{h} \backslash \bar{\Omega}} .
$$

The first term in the right-hand side of the inequality in the previous lemma is directly bounded by Lemma 4.1. In what follows we give estimates for the remaining four terms. We begin with the density term in the following lemma:

Lemma 4.3. There exists a positive constant $C$ such that

$$
\inf _{v_{h} \in \mathcal{G}_{h}}\left\|\hat{u}-v_{h}\right\|_{\operatorname{div}, \Omega_{h}} \leq C h^{r_{h}^{\prime}}\|f\|_{0, \mathbb{R}^{2}} .
$$

Proof. Let $R \hat{u} \in \mathcal{R}_{h}\left(\Omega_{h}\right)$ be the Raviart-Thomas interpolant of $\left.\hat{u}\right|_{\Omega_{h}}$, with its degrees of freedom defined by (3.14). Since $\hat{u} \in \mathrm{H}^{r_{h}^{\prime}}\left(\widetilde{\Omega}_{h}\right)$, then $R \hat{u}$ is well defined. However, in general, $R \hat{u} \notin \mathcal{V}_{h}$ because $\hat{u} \cdot \nu_{h}$ does not necessarily vanish on the edges $s_{T}$ corresponding to those boundary triangles $T \in \mathcal{T}_{h}^{\partial}$ with $T \subset \widetilde{T}$ (see Fig. 2).

Then, to define an "interpolant" $\widetilde{R} \hat{u} \in \mathcal{V}_{h}$, we modify the degrees of freedom of $R \hat{u}$ by explicitly imposing $\widetilde{R} \hat{u} \cdot \nu_{h}=0$ on these edges. Namely, let $\widetilde{R} \hat{u}$ be the unique vector field in $\mathcal{R}_{h}\left(\Omega_{h}\right)$ satisfying

$$
\left.\widetilde{R} \hat{u} \cdot \nu_{\ell}\right|_{\ell}= \begin{cases}0, & \text { if } \ell=s_{t} \text { for } T \in \mathcal{T}_{h}^{\partial}: T \subset \widetilde{T}, \\ \left.R \hat{u} \cdot \nu_{\ell}\right|_{\ell}, & \text { otherwise, }\end{cases}
$$

where $\nu_{\ell}$ denotes a unit normal to $\ell$. Then, $\widetilde{R} \hat{u} \in \mathcal{V}_{h}$.

In what follows, we bound the difference between this modified RaviartThomas interpolant and the standard one:

$$
\|\widetilde{R} \hat{u}-R \hat{u}\|_{\operatorname{div}, \Omega_{h}}^{2}=\sum_{T \in \mathcal{T}_{h}}\|\widetilde{R} \hat{u}-R \hat{u}\|_{\operatorname{div}, T}^{2}=\sum_{T \in \mathcal{T}_{h}^{\partial}: T \subset \widetilde{T}}\|\widetilde{R} \hat{u}-R \hat{u}\|_{\operatorname{div}, T}^{2} .
$$

Let $T \in \mathcal{T}_{h}^{\partial}$ such that $T \subset \widetilde{T}$; by definition we have

$$
\left.(\widetilde{R} \hat{u}-R \hat{u})\right|_{T}=\left(\frac{1}{\left|s_{T}\right|} \int_{s_{T}} \hat{u} \cdot \nu_{h} d s\right) \phi_{s_{T}},
$$

where $\phi_{s_{T}}$ is the standard basis function of $\mathcal{R} \mathcal{T}_{0}(T)$ associated to the edge $s_{T}$. Hence, straightforward computations yield

$$
\|\widetilde{R} \hat{u}-R \hat{u}\|_{\operatorname{div}, T}=\left|\frac{1}{\left|s_{T}\right|} \int_{s_{T}} \hat{u} \cdot \nu_{h} d s\right|\left\|\phi_{s_{T}}\right\|_{\operatorname{div}, T} \leq \frac{C}{\left|s_{T}\right|}\left|\int_{s_{T}} \hat{u} \cdot \nu_{h} d s\right| .
$$


On the other hand, since $\int_{\sigma_{T}} \hat{u} \cdot \nu d s=0$, by using Lemma 3.2 we have

$\int_{s_{T}} \hat{u} \cdot \nu_{h} d s=\int_{\omega_{T}} \operatorname{div} \hat{u} d x \leq\left|\omega_{T}\right|^{1 / 2}\|\operatorname{div} \hat{u}\|_{0, \omega_{T}} \leq C h_{T}^{3 / 2}\|\operatorname{div} \hat{u}\|_{0, \omega_{T}}$, and since $\left|s_{T}\right| \geq C h_{T}$,

$$
\|\widetilde{R} \hat{u}-R \hat{u}\|_{\operatorname{div}, T} \leq C h_{T}^{1 / 2}\|\operatorname{div} \hat{u}\|_{0, \widetilde{T} \backslash T} .
$$

Hence,

$$
\|\widetilde{R} \hat{u}-R \hat{u}\|_{\operatorname{div}, \Omega_{h}}^{2} \leq \sum_{T \in \mathcal{T}_{h}^{\partial}: T \subset \widetilde{T}} C h_{T}\|\operatorname{div} \hat{u}\|_{0, \widetilde{T} \backslash T}^{2} \leq C h\|\operatorname{div} \hat{u}\|_{0, \Omega \backslash \bar{\Omega}_{h}}^{2} .
$$

Then, because of (4.8), Lemma 3.3 yields

$$
\|\widetilde{R} \hat{u}-R \hat{u}\|_{\operatorname{div}, \Omega_{h}} \leq C h^{3 / 2}\|\operatorname{div} \hat{u}\|_{1, \widetilde{\Omega}_{h}} \leq C h^{3 / 2}\|f\|_{0, \mathbb{R}^{2}} .
$$

Hence, we use (3.16), (3.17), (4.7), and (4.8), to obtain

$$
\begin{aligned}
\|\hat{u}-\widetilde{R} \hat{u}\|_{\operatorname{div}, \Omega_{h}} & \leq\|\hat{u}-R \hat{u}\|_{\operatorname{div}, \Omega_{h}}+\|R \hat{u}-\widetilde{R} \hat{u}\|_{\operatorname{div}, \Omega_{h}} \\
& \leq C h^{r_{h}^{\prime}}\left(\|\hat{u}\|_{r_{h}^{\prime}, \widetilde{\Omega}_{h}}+\|\operatorname{div} \hat{u}\|_{r_{h}^{\prime}, \widetilde{\Omega}_{h}}\right)+C h^{3 / 2}\|f\|_{0, \mathbb{R}^{2}} \\
& \leq C h^{r_{h}^{\prime}}\|f\|_{0, \mathbb{R}^{2}} .
\end{aligned}
$$

Now, let $P: \mathcal{V}_{h} \longrightarrow \mathcal{K}_{h}$ be the $\mathrm{H}\left(\operatorname{div}, \Omega_{h}\right)$-orthogonal projection and let $v_{h}:=\widetilde{R} \hat{u}-P(\widetilde{R} \hat{u}) \in \mathcal{G}_{h}$. Then $v_{h}$ and $P(\widetilde{R} \hat{u}) \in \mathcal{K}_{h}$ are $\mathrm{H}\left(\operatorname{div}, \Omega_{h}\right)$ orthogonal. On the other hand, since $\hat{u} \in \mathcal{G}\left(\widetilde{\Omega}_{h}\right)$, then $\left.\hat{u}\right|_{\Omega_{h}}$ and $P(\widetilde{R} \hat{u})$ are also $\mathrm{H}\left(\operatorname{div}, \Omega_{h}\right)$-orthogonal. Hence

$$
\begin{aligned}
\left\|\hat{u}-v_{h}\right\|_{\operatorname{div}, \Omega_{h}}^{2} & \leq\left\|\left(\hat{u}-v_{h}\right)\right\|_{\operatorname{div}, \Omega_{h}}^{2}+\|P(\widetilde{R} \hat{u})\|_{\operatorname{div}, \Omega_{h}}^{2} \\
& =\left\|\left(\hat{u}-v_{h}\right)-P(\widetilde{R} \hat{u})\right\|_{\operatorname{div}, \Omega_{h}}^{2} \\
& =\|\hat{u}-\widetilde{R} \hat{u}\|_{\operatorname{div}, \Omega_{h}}^{2} .
\end{aligned}
$$

Therefore, we conclude the proof by combining the two previous inequalities.

Now we deal with the consistency term in Lemma 4.2:

Lemma 4.4. There exists a positive constant $C$ such that

$$
\int_{\Omega_{h}} \operatorname{div}\left(\hat{u}-u_{h}\right) \operatorname{div} w_{h} d x \leq C h^{r_{h}^{\prime}}\|f\|_{0, \mathbb{R}^{2}}\left\|w_{h}\right\|_{\operatorname{div}, \Omega_{h}} \quad \forall w_{h} \in \mathcal{G}_{h} .
$$


Proof. Let $w_{h} \in \mathcal{G}_{h}$. Consider the Helmholtz decomposition $\bar{w}_{h}=\nabla \xi+\chi$ with $\nabla \xi \in \mathcal{G}\left(\widetilde{\Omega}_{h}\right)$ and $\chi \in \mathcal{K}\left(\widetilde{\Omega}_{h}\right)$ as in Lemma 3.4. Then, using (3.22) and (4.3), and integrating by parts, we have

$$
\begin{aligned}
\int_{\Omega_{h}} & \operatorname{div}\left(\hat{u}-u_{h}\right) \operatorname{div} w_{h} d x \\
& =\int_{\widetilde{\Omega}_{h}} \operatorname{div} \hat{u} \operatorname{div} \bar{w}_{h} d x-\int_{\Omega_{h}} \operatorname{div} u_{h} \operatorname{div} w_{h} d x \\
& =\int_{\widetilde{\Omega}_{h}} \operatorname{div} \hat{u} \operatorname{div}(\nabla \xi) d x-\int_{\Omega_{h}} f \cdot w_{h} d x \\
& =\int_{\widetilde{\Omega}_{h}}\left[(\operatorname{div} u)^{e}+\hat{c}\right] \operatorname{div}(\nabla \xi) d x-\int_{\Omega_{h}} f \cdot \nabla \xi d x-\int_{\Omega_{h}} f \cdot \chi d x \\
& =-\int_{\widetilde{\Omega}_{h}} \nabla\left[(\operatorname{div} u)^{e}\right] \cdot \nabla \xi d x-\int_{\Omega_{h}} f \cdot \nabla \xi d x-\int_{\Omega_{h}} f \cdot \chi d x .
\end{aligned}
$$

Hence, using (3.9) we obtain

$$
\text { (4.10) } \begin{aligned}
\int_{\Omega_{h}} \operatorname{div}\left(\hat{u}-u_{h}\right) \operatorname{div} w_{h} d x= & -\int_{\Omega_{h} \backslash \bar{\Omega}} \nabla(\operatorname{div} u)^{e} \cdot \nabla \xi d x \\
& +\int_{\Omega_{\backslash \bar{\Omega}_{h}}} f \cdot \nabla \xi d x \\
& -\int_{\Omega_{h} \backslash \bar{\Omega}} f \cdot \nabla \xi d x-\int_{\Omega_{h}} f \cdot \chi d x .
\end{aligned}
$$

In what follows, we estimate the four terms in the right side above. For the first one we use Lemma 3.3, (4.2), (3.10), and (3.19) to obtain

$$
\begin{aligned}
\left|\int_{\Omega_{h} \backslash \bar{\Omega}} \nabla(\operatorname{div} u)^{e} \cdot \nabla \xi d x\right| & \leq\left\|(\operatorname{div} u)^{e}\right\|_{1, \Omega_{h} \backslash \bar{\Omega}} C h^{r_{h}^{\prime}}\|\nabla \xi\|_{r_{h}^{\prime}, \widetilde{\Omega}_{h}} \\
& \leq C h^{r_{h}^{\prime}}\|\operatorname{div} u\|_{1, \Omega}\left\|\operatorname{div} w_{h}\right\|_{0, \Omega_{h}} \\
& \leq C h^{r_{h}^{\prime}}\|f\|_{0, \mathbb{R}^{2}}\left\|w_{h}\right\|_{\operatorname{div}, \Omega_{h}} .
\end{aligned}
$$

Analogously, for the next two terms, by using Lemma 3.3 and (3.19) we have

$$
\left|\int_{\Omega \backslash \bar{\Omega}_{h}} f \cdot \nabla \xi d x\right|+\left|\int_{\Omega_{h} \backslash \bar{\Omega}} f \cdot \nabla \xi d x\right| \leq C h^{r_{h}^{\prime}}\|f\|_{0, \mathbb{R}^{2}}\left\|w_{h}\right\|_{\operatorname{div}, \Omega_{h}} .
$$

For the last term, we use Cauchy-Schwarz inequality and (3.20) to obtain

$$
\left|\int_{\Omega_{h}} f \cdot \chi d x\right| \leq\|f\|_{0, \Omega_{h}}\|\chi\|_{0, \Omega_{h}} \leq C h^{r_{h}^{\prime}}\|f\|_{0, \mathbb{R}^{2}}\left\|w_{h}\right\|_{\operatorname{div}, \Omega_{h}} .
$$

Thus, we conclude the proof. 
It only remains to estimate the last two terms in Lemma 4.2. We do it in the following two lemmas:

Lemma 4.5. There exists a positive constant $C$ such that

$$
\|u\|_{\operatorname{div}, \Omega \backslash \bar{\Omega}_{h}} \leq C h^{r}\|f\|_{0, \mathbb{R}^{2}} .
$$

Proof. Because $u \in \mathrm{H}^{r}(\Omega)^{2}$ and $\operatorname{div} u \in \mathrm{H}^{1}(\Omega)$, we use Lemma 3.3 and estimates (3.8) and (3.10) to obtain:

$$
\begin{aligned}
& \|u\|_{0, \Omega \backslash \bar{\Omega}_{h}} \leq C h^{r}\|u\|_{r, \Omega} \leq C h^{r}\|f\|_{0, \mathbb{R}^{2}} \\
& \|\operatorname{div} u\|_{0, \Omega \backslash \bar{\Omega}_{h}} \leq C h\|\operatorname{div} u\|_{1, \Omega} \leq C h\|f\|_{0, \mathbb{R}^{2}} .
\end{aligned}
$$

Lemma 4.6. There exists a positive constant $C$ such that

$$
\|\hat{u}\|_{\operatorname{div}, \Omega_{h} \backslash \bar{\Omega}} \leq C h^{r_{h}^{\prime}}\|f\|_{0, \mathbb{R}^{2}} .
$$

Proof. Because $\hat{u} \in \mathrm{H}^{r_{h}^{\prime}}\left(\widetilde{\Omega}_{h}\right)^{2}$, we use Lemma 3.3 and (4.7) to obtain

$$
\|\hat{u}\|_{0, \Omega_{h} \backslash \bar{\Omega}} \leq C h^{r_{h}^{\prime}}\|\hat{u}\|_{r_{h}^{\prime}, \widetilde{\Omega}_{h}} \leq C h^{r_{h}^{\prime}}\|f\|_{0, \mathbb{R}^{2}} .
$$

On the other hand, because of (4.3), $\|\operatorname{div} \hat{u}\|_{0, \Omega_{h} \backslash \bar{\Omega}}=\left\|(\operatorname{div} u)^{e}+\hat{c}\right\|_{0, \Omega_{h} \backslash \bar{\Omega}}$. Then, we use Lemma 3.3, (4.5), Lemma 3.2, (4.2), and (3.10) to obtain

$$
\begin{aligned}
\|\operatorname{div} \hat{u}\|_{0, \Omega_{h} \backslash \bar{\Omega}} & \leq\left\|(\operatorname{div} u)^{e}\right\|_{0, \Omega_{h} \backslash \bar{\Omega}}+|\hat{c}|\left|\Omega_{h} \backslash \bar{\Omega}\right|^{1 / 2} \\
& \leq C h\left\|(\operatorname{div} u)^{e}\right\|_{1, \widetilde{\Omega}_{h}}+C h^{3}\|f\|_{0, \mathbb{R}^{2}} \\
& \leq C h\|f\|_{0, \mathbb{R}^{2}} \cdot \quad \square
\end{aligned}
$$

Now we may conclude the convergence in norm of $A_{h}$ to $A$ :

Lemma 4.7. There exists a positive constant $C$ such that

$$
\left\|\left(A-A_{h}\right) f\right\|_{\operatorname{div}, \mathbb{R}^{2}} \leq C h^{r_{h}^{\prime}}\|f\|_{0, \mathbb{R}^{2}} \quad \forall f \in \mathrm{L}^{2}\left(\mathbb{R}^{2}\right)^{2} .
$$

Proof. It is an immediate consequence of the previous six lemmas.

By virtue of the previous lemma, we are able to apply the spectral approximation theory for compact operators (see [2]) to obtain error estimates for the eigenfunctions.

Let $\mu>0$ be a fixed eigenvalue of $A$ with algebraic multiplicity $m$. Let $\mathcal{E}$ be the corresponding associated eigenspace. Since Lemma $4.7 \mathrm{im}-$ plies that $\left\|A-A_{h}\right\|_{\mathrm{H}\left(\mathrm{div}, \mathbb{R}^{2}\right)} \rightarrow 0$ as $h \rightarrow 0$, then there exist exactly $m$ eigenvalues of $A_{h}, \mu_{h}^{(1)}, \ldots, \mu_{h}^{(m)}$ (repeated accordingly to their respective multiplicities) converging to $\mu$ (see [26]). Let $\mathcal{E}_{h}$ be the direct sum of the corresponding associated eigenspaces. 
We recall the definition of the gap $\widehat{\delta}$ between two closed subspaces, $\mathcal{Y}$ and $\mathcal{Z}$, of $\mathrm{H}\left(\operatorname{div}, \mathbb{R}^{2}\right)$ :

$$
\widehat{\delta}(\mathcal{Y}, \mathcal{Z}):=\max \{\delta(\mathcal{Y}, \mathcal{Z}), \delta(\mathcal{Z}, \mathcal{Y})\}
$$

with

$$
\delta(\mathcal{Y}, \mathcal{Z}):=\sup _{\substack{v \in \mathcal{Y} \\\|v\|_{\operatorname{div}, \mathbb{R}^{2}}=1}}\left(\inf _{w \in \mathcal{Z}}\|v-w\|_{\operatorname{div}, \mathbb{R}^{2}}\right) .
$$

Then, the following error estimate holds:

Theorem 4.1. There exist a positive constant $C$ such that

$$
\widehat{\delta}\left(\mathcal{E}_{h}, \mathcal{E}\right) \leq C h^{r_{h}^{\prime}}
$$

with $r_{h}^{\prime}$ as defined in Remark 3.1.

Proof. It is an immediate consequence of Theorem 7.1 in [2] and Lemma 4.7.

In order to prove a double-order error estimate for the approximate eigenvalues, we will use the following lemma:

Lemma 4.8. There exist a positive constant $C$ such that

$$
\left|\int_{\widetilde{\Omega}_{h}}\left(A-A_{h}\right) f \cdot g d x\right| \leq C h^{2 r_{h}^{\prime}}\|f\|_{0, \mathbb{R}^{2}}\|g\|_{0, \mathbb{R}^{2}} \quad \forall f, g \in \mathcal{E} .
$$

Proof. Let $f, g \in \mathcal{E}$. We denote

$$
\begin{array}{llll}
\bar{u}=A f, & u=\left.\bar{u}\right|_{\Omega}, & \bar{u}_{h}=A_{h} f, & u_{h}=\left.\bar{u}_{h}\right|_{\Omega_{h}}, \\
\bar{v}=A g, & v=\left.\bar{v}\right|_{\Omega}, & \bar{v}_{h}=A_{h} g, & v_{h}=\left.\bar{v}_{h}\right|_{\Omega_{h}} .
\end{array}
$$

We have

$$
\begin{aligned}
\int_{\widetilde{\Omega}_{h}} & \left(A-A_{h}\right) f \cdot g d x=\int_{\widetilde{\Omega}_{h}}\left(\bar{u}-\bar{u}_{h}\right) \cdot g d x \\
= & \int_{\Omega} \operatorname{div} u \operatorname{div} v d x-\int_{\Omega_{h}} \operatorname{div} u_{h} \operatorname{div} v_{h} d x \\
= & \int_{\Omega} \operatorname{div}\left(u-\bar{u}_{h}\right) \operatorname{div}\left(v-\bar{v}_{h}\right) d x+\int_{\Omega_{\cap} \Omega_{h}} \operatorname{div}\left(u-u_{h}\right) \operatorname{div} v_{h} d x \\
& +\int_{\Omega \cap \Omega_{h}} \operatorname{div} u_{h} \operatorname{div}\left(v-v_{h}\right) d x-\int_{\Omega_{h} \backslash \bar{\Omega}} \operatorname{div} u_{h} \operatorname{div} v_{h} d x
\end{aligned}
$$


In what follows we estimate the terms in the right side of the above equation. Because of Cauchy-Schwarz inequality and Lemma 4.7, we have

$$
\int_{\Omega} \operatorname{div}\left(u-\bar{u}_{h}\right) \operatorname{div}\left(v-\bar{v}_{h}\right) d x \leq C h^{2 r_{h}^{\prime}}\|f\|_{0, \mathbb{R}^{2}}\|g\|_{0, \mathbb{R}^{2}} .
$$

The next two terms are similar (changing the role of the functions $u, u_{h}$, and $v_{h}$, by $v, v_{h}$, and $u_{h}$, respectively). Then we write down the estimate only for the first one.

Since $\bar{u}=A f \in \mathcal{E}$, because of (3.11), $\operatorname{div} u \in \mathrm{H}^{1+r}(\Omega)$. Then, there is an extension $(\operatorname{div} u)^{e} \in \mathrm{H}^{1+r}\left(\mathbb{R}^{2}\right)$ satisfying (4.1) for $s=1+r$; namely,

$$
\left\|(\operatorname{div} u)^{e}\right\|_{1+r, \mathbb{R}^{2}} \leq C\|\operatorname{div} u\|_{1+r, \Omega} \leq C\|f\|_{0, \mathbb{R}^{2}} .
$$

Let $\hat{u}=\nabla \hat{\varphi} \in \mathcal{G}\left(\widetilde{\Omega}_{h}\right)$. with $\hat{\varphi}$ being the solution of (4.3)-(4.4). Then we have

$$
\begin{aligned}
\int_{\Omega \cap \Omega_{h}} & \operatorname{div}\left(u-u_{h}\right) \operatorname{div} v_{h} d x \\
= & \int_{\Omega_{h}}\left[(\operatorname{div} u)^{e}-\operatorname{div} u_{h}\right] \operatorname{div} v_{h} d x-\int_{\Omega_{h} \backslash \bar{\Omega}}(\operatorname{div} u)^{e} \operatorname{div} v_{h} d x \\
& +\int_{\Omega_{h} \backslash \bar{\Omega}} \operatorname{div} u_{h} \operatorname{div} v_{h} d x \\
= & \int_{\Omega_{h}}\left[(\operatorname{div} u)^{e}-\operatorname{div} \hat{u}\right] \operatorname{div} v_{h} d x+\int_{\Omega_{h}}\left(\operatorname{div} \hat{u}-\operatorname{div} u_{h}\right) \operatorname{div} v_{h} d x \\
& -\int_{\Omega_{h} \backslash \bar{\Omega}}(\operatorname{div} u)^{e} \operatorname{div} v_{h} d x+\int_{\Omega_{h} \backslash \bar{\Omega}} \operatorname{div} u_{h} \operatorname{div} v_{h} d x \\
= & \hat{c} \int_{\Omega_{h}} \operatorname{div} v_{h} d x+\int_{\Omega_{h}} \operatorname{div}\left(\hat{u}-u_{h}\right) \operatorname{div} v_{h} d x \\
& -\int_{\Omega_{h} \backslash \bar{\Omega}}(\operatorname{div} u)^{e} \operatorname{div} v_{h} d x+\int_{\Omega_{h} \backslash \bar{\Omega}} \operatorname{div} u_{h} \operatorname{div} v_{h} d x
\end{aligned}
$$

Now, let $\bar{v}_{h}=\nabla \xi+\chi$ as in Lemma 3.4. The second term in the right hand side of this equation can be dealt with by repeating the argument in the proof of Lemma 4.4 (see (4.10)). Then we obtain

$$
\begin{aligned}
\int_{\Omega \cap \Omega_{h}} \operatorname{div}\left(u-u_{h}\right) \operatorname{div} v_{h} d x \\
=\hat{c} \int_{\Omega_{h}} \operatorname{div} v_{h} d x-\int_{\Omega_{h} \backslash \bar{\Omega}} \nabla(\operatorname{div} u)^{e} \cdot \nabla \xi d x \\
\quad+\int_{\Omega \backslash \bar{\Omega}_{h}} f \cdot \nabla \xi d x-\int_{\Omega \cap \Omega_{h}} f \cdot \chi d x \\
\quad-\int_{\Omega_{h} \backslash \bar{\Omega}}(\operatorname{div} u)^{e} \operatorname{div} v_{h} d x+\int_{\Omega_{h} \backslash \bar{\Omega}} \operatorname{div} u_{h} \operatorname{div} v_{h} d x
\end{aligned}
$$


where we have used that $\left.f\right|_{\Omega_{h} \backslash \bar{\Omega}}=0$. Notice that the last integral also appears in (4.11). Thus, to conclude the lemma, it is enough to estimate each term in the right-hand side of the above equation.

Because of (4.5) and (3.23), we have

$$
\left|\hat{c} \int_{\Omega_{h}} \operatorname{div} v_{h} d x\right| \leq C h^{2}\|f\|_{0, \mathbb{R}^{2}}\left\|v_{h}\right\|_{\operatorname{div}, \Omega_{h}} \leq C h^{2}\|f\|_{0, \mathbb{R}^{2}}\|g\|_{0, \mathbb{R}^{2}} .
$$

For the second term we use Lemma 3.3, (4.12), (3.19), and (3.23) to obtain

$$
\begin{aligned}
\left|\int_{\Omega_{h} \backslash \bar{\Omega}} \nabla(\operatorname{div} u)^{e} \cdot \nabla \xi d x\right| & \leq C h^{r}\left\|(\operatorname{div} u)^{e}\right\|_{1+r, \widetilde{\Omega}_{h}} C h^{r_{h}^{\prime}}\|\nabla \xi\|_{r_{h}^{\prime}, \widetilde{\Omega}_{h}} \\
& \leq C h^{r+r_{h}^{\prime}}\|f\|_{0, \mathbb{R}^{2}}\|g\|_{0, \mathbb{R}^{2}} .
\end{aligned}
$$

To estimate the third and forth terms, we use that $\bar{u}=A f=\mu f$ because $f \in \mathcal{E}$. Then $f=\frac{1}{\mu} u$ in $\Omega$. Hence, by using Lemma 3.3, (3.8), (3.19), and (3.23) we have

$$
\begin{aligned}
& \left|\int_{\Omega \backslash \bar{\Omega}_{h}} f \cdot \nabla \xi d x\right| \leq \frac{C h^{r}}{\mu}\|u\|_{r, \Omega} C h^{r_{h}^{\prime}}\|\nabla \xi\|_{r_{h}^{\prime}, \widetilde{\Omega}_{h}} \\
& \leq C h^{r+r_{h}^{\prime}}\|f\|_{0, \mathbb{R}^{2}}\|g\|_{0, \mathbb{R}^{2}} .
\end{aligned}
$$

Regarding the forth term we have

$$
\begin{aligned}
& \int_{\Omega \cap \Omega_{h}} f \cdot \chi d x=\frac{1}{\mu}\left[\int_{\Omega \cap \Omega_{h}}(u-\hat{u}) \cdot \chi d x+\int_{\Omega \cap \Omega_{h}} \hat{u} \cdot \chi d x\right] \\
& \quad=\frac{1}{\mu}\left[\int_{\Omega \cap \Omega_{h}}(u-\hat{u}) \cdot \chi d x-\int_{\Omega \backslash \bar{\Omega}_{h}} \hat{u} \cdot \chi d x-\int_{\Omega_{h} \backslash \bar{\Omega}} \hat{u} \cdot \chi d x\right],
\end{aligned}
$$

the latter because $\int_{\widetilde{\Omega}_{h}} u \cdot \chi=0$, from the orthogonality of $\mathcal{G}\left(\widetilde{\Omega}_{h}\right)$ and $\mathcal{K}\left(\widetilde{\Omega}_{h}\right)$. Then, by using Cauchy-Schwarz inequality, Lemmas 4.1 and 3.3, (3.20), (3.23), and (4.7) we obtain

$$
\begin{aligned}
\left|\int_{\Omega \cap \Omega_{h}} f \cdot \chi d x\right| \leq & C h^{2 r_{h}^{\prime}}\|f\|_{0, \mathbb{R}^{2}} C h^{r_{h}^{\prime}}\left\|v_{h}\right\|_{\operatorname{div}, \Omega_{h}} \\
& +2 C h^{r_{h}^{\prime}}\|\hat{u}\|_{r_{h}^{\prime}, \widetilde{\Omega}_{h}} C h^{r_{h}^{\prime}}\left\|v_{h}\right\|_{\operatorname{div}, \Omega_{h}} \\
\leq & C h^{2 r_{h}^{\prime}}\|f\|_{0, \mathbb{R}^{2}}\|g\|_{0, \mathbb{R}^{2} .}
\end{aligned}
$$

Finally, to estimate the last two terms notice that, because of Lemma 4.7,

$$
\left\|v_{h}\right\|_{\operatorname{div}, \Omega_{h} \backslash \bar{\Omega}}=\left\|v_{h}-\bar{v}\right\|_{\operatorname{div}, \Omega_{h} \backslash \bar{\Omega}} \leq\left\|\bar{v}_{h}-\bar{v}\right\|_{\operatorname{div}, \mathbb{R}^{2}} \leq C h^{r_{h}^{\prime}}\|g\|_{0, \mathbb{R}^{2}}
$$


and, analogously, $\left\|u_{h}\right\|_{\operatorname{div}, \Omega_{h} \backslash \bar{\Omega}} \leq C h^{r_{h}^{\prime}}\|f\|_{0, \mathbb{R}^{2}}$. Then, by using Lemma 3.3 and (4.12) we have

$$
\begin{aligned}
& \left|\int_{\Omega_{h} \backslash \bar{\Omega}}(\operatorname{div} u)^{e} \operatorname{div} v_{h} d x\right| \leq C h\left\|(\operatorname{div} u)^{e}\right\|_{1, \widetilde{\Omega}_{h}}\left\|v_{h}\right\|_{\operatorname{div}, \Omega_{h} \backslash \bar{\Omega}} \\
& \leq C h^{1+r_{h}^{\prime}}\|f\|_{0, \mathbb{R}^{2}}\|g\|_{0, \mathbb{R}^{2}}
\end{aligned}
$$

and

$$
\begin{aligned}
\left|\int_{\Omega_{h} \backslash \bar{\Omega}} \operatorname{div} u_{h} \operatorname{div} v_{h} d x\right| & \leq\left\|u_{h}\right\|_{\operatorname{div}, \Omega_{h} \backslash \bar{\Omega}}\left\|v_{h}\right\|_{\operatorname{div}, \Omega_{h} \backslash \bar{\Omega}} \\
& \leq C h^{2 r_{h}^{\prime}}\|f\|_{0, \mathbb{R}^{2}}\|g\|_{0, \mathbb{R}^{2} .}
\end{aligned}
$$

Thus, we conclude the proof.

Now we are able to establish a double-order error estimate for the approximate eigenvalues. We know that $\lambda=1 / \mu$ is a positive eigenvalue of problem (2.1) with multiplicity $m$, and $\lambda_{h}^{(i)}=1 / \mu_{h}^{(i)}, i=1, \ldots, m$, are the eigenvalues of problem (2.2) converging to $\lambda$. Then, the following error estimate holds:

Theorem 4.2. There exist a positive constant $C$ such that

$$
\left|\lambda-\lambda_{h}^{(i)}\right| \leq C h^{2 r_{h}^{\prime}}, \quad i=1, \ldots, m .
$$

Proof. It is an immediate consequence of the previous lemma, Lemma 4.7 and Remark 7.5 in [2].

\section{Numerical Examples}

In this section we report the results obtained on two numerical tests which confirm our theoretical statements. One of the tests corresponds to a domain with a smooth boundary and the other to a domain with reentrant corners.

In both tests, we have computed a few eigenvalues on several differently refined meshes in order to estimate the respective orders of convergence. Fig. 3 shows the domains and corresponding coarse meshes for each test.

The results obtained for the annular domain of the first test are reported in Table 1 . In this case, all the eigenvalues have multiplicity 2 . This table shows the approximations of the smallest three double eigenvalues computed on some of the used meshes. Each mesh is identified by its total number of degrees of freedom (d.o.f.). We also include for each eigenvalue the estimated order of convergence and an extrapolated more accurate approximation, both obtained by means of a least square fitting of the computed 
Test 1: Domain with a $\mathcal{C}^{\infty}$-boundary

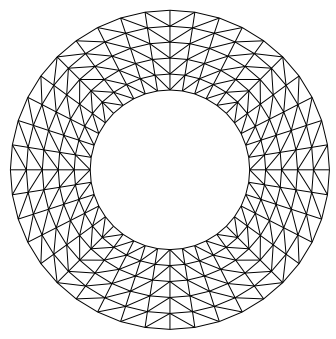

Test 2: Domain with reentrant corners

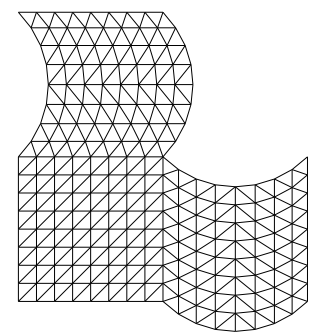

Fig. 3. Domains and meshes.

Table 1. Test 1: Numerical results for a domain with a $\mathcal{C}^{\infty}$-boundary.

\begin{tabular}{ccccccc}
\hline d.o.f. & 2480 & 9760 & 21840 & 38720 & Order & Extrapolated \\
\hline$\lambda_{h 1}^{(1)}$ & 1.835302 & 1.835177 & 1.835154 & 1.835146 & 2.03 & 1.835136 \\
$\lambda_{h 1}^{(2)}$ & 1.835302 & 1.835177 & 1.835154 & 1.835146 & 2.03 & 1.835136 \\
$\lambda_{h 2}^{(1)}$ & 7.189809 & 7.189092 & 7.188958 & 7.188915 & 2.02 & 7.188856 \\
$\lambda_{h 2}^{(2)}$ & 7.191344 & 7.189473 & 7.189132 & 7.189011 & 2.02 & 7.188861 \\
$\lambda_{h 3}^{(1)}$ & 15.674033 & 15.666375 & 15.664954 & 15.664458 & 2.00 & 15.663820 \\
$\lambda_{h 3}^{(2)}$ & 15.674033 & 15.666375 & 15.664954 & 15.664458 & 2.00 & 15.663820
\end{tabular}

values. The rate of convergence (in powers of $h$ ) is clearly 2 , which agrees with the fact that, in this case, $r=1$.

Table 2 shows the corresponding results obtained for the curved Lshaped domain of the second test. In this case, the domain has two reentrant corners of sizes $5 \pi / 4$ and $3 \pi / 2$. Because of this, some of the eigenvalues are computed with an order clearly smaller than 2 (in powers of $h$ ). Indeed, in this case $r=2 / 3$ and then the error estimates proved in the previous section is $\left|\lambda_{i}-\lambda_{h i}\right| \leq C h^{4 / 3}$. It can be seen that this order of convergence is practically attained for the two first eigenvalues.

Table 2. Test 2: Numerical results for a domain with a reentrant corner

\begin{tabular}{ccccccc}
\hline d.o.f. & 3680 & 14560 & 32640 & 57920 & Order & Extrapolated \\
\hline$\lambda_{h 1}$ & 1.359828 & 1.362873 & 1.363690 & 1.364048 & 1.37 & 1.364792 \\
$\lambda_{h 2}$ & 3.349844 & 3.351317 & 3.351717 & 3.351892 & 1.36 & 3.352258 \\
$\lambda_{h 3}$ & 9.558594 & 9.562825 & 9.563608 & 9.563884 & 2.00 & 9.564235 \\
$\lambda_{h 4}$ & 9.568797 & 9.571612 & 9.572166 & 9.572368 & 1.90 & 9.572643 \\
$\lambda_{h 5}$ & 11.035413 & 11.038415 & 11.039169 & 11.039485 & 1.49 & 11.040076
\end{tabular}


Acknowledgment. The authors thanks the anonymous referees for their valuable suggestions and Monique Dauge for the personal communication [16].

\section{References}

1. A. Alonso, A. Dello Russo, and V. Vampa: A posteriori error estimates in finite element acoustic analysis. J. Comput. Appl. Math. 117 (2000) 105-119.

2. I. Babuška and J. Osborn: Eigenvalue problems. In: Handbook of Numerical Analysis. Vol II. P.G. Ciarlet and J.L. Lions, eds. North Holland, Amsterdam, 1991, pp. 641-787.

3. A. Bermúdez, R. Durán, M.A. Muschietti, R. Rodríguez, and J. Solomin: Finite element vibration analysis of fluid-solid systems without spurious modes. SIAM J. Numer. Anal. 32 (1995) 1280-1295.

4. A. Bermúdez, R. Durán, and R. Rodríguez: Finite element solution of incompressible fluid-structure vibration problems. Internat. J. Numer. Methods Eng. 40 (1997) 14351448.

5. A. Bermúdez, R. Durán, and R. Rodríguez: Finite element analysis of compressible and incompressible fluid-solid systems. Math. Comp. 67 (1998) 111-136.

6. A. Bermúdez, R. Durán, R. Rodríguez, and J. Solomin: Finite element analysis of a quadratic eigenvalue problem arising in dissipative acoustics. SIAM J. Numer. Anal. 38 (2000) 267-291.

7. A. Bermúdez, L. Hervella-Nieto, and R. Rodríguez: Finite element computation of the vibrations of a plate-fluid system with interface damping. Comput. Methods Appl. Mech. Eng. 190 (2001) 3021-3038.

8. A. Bermúdez and R. Rodríguez: Finite element computation of the vibration modes of a fluid-solid system. Comput. Methods Appl. Mech. Eng. 119 (1994) 355-370.

9. A. Bermúdez, R. Rodríguez: Modelling and numerical solution of elastoacoustic vibrations with interface damping. Internat. J. Numer. Methods Eng. 46 (1999) 1763-1779.

10. D. Boffi, F. Brezzi, and L. Gastaldi: On the convergence of eigenvalues for mixed formulations. Ann. Scuola Norm. Sup. Pisa 25 (1997) 131-154.

11. D. Boffi, F. Brezzi, and L. Gastaldi: On the problem of spurious eigenvalues in the approximation of linear elliptic problems in mixed form. Math. Comp. 69 (2000) 121140.

12. S. Brenner, L. R. Scott: Mixed and Hybrid Finite Element Methods, Springer, New York, Berlin, Heidelberg, 1996

13. H.C. Chen and R.L. Taylor: Vibration analysis of fluid-solid systems using a finite element displacement formulation. Internat. J. Numer. Methods Eng. 29 (1990) 683698.

14. P.G. Ciarlet: Basic error estimates for elliptic problems. In: Handbook of Numerical Analysis. Vol. II. P.G. Ciarlet and J.L. Lions, eds. North Holland, Amsterdam, 1991, pp. 17-351.

15. C. Conca, J. Planchard, and M. Vanninathan: Fluids and Periodic Structures. Masson, Paris, 1992.

16. M. Costabel, M. Dauge, and A. McIntosh: Personal communication.

17. R.A. Devore and R.C. Sharpley: Besov spaces on domains in $\mathbb{R}^{d}$. Trans. Amer. Math. Soc. 335 (1993) 843-864.

18. R.G. Durán, L. Gastaldi, and C. Padra: A posteriori error estimators for mixed approximations of eigenvalue problems. Math. Models Methods Appl. Sci. 9 (1999) 11651178. 
19. R. Durán, L. Hervella-Nieto, E. Liberman, R. Rodríguez, and J. Solomin: Finite element analysis of the vibration problem of a plate coupled with a fluid. Numer. Math. 86 (2000) 591-616.

20. M. Feistauer and A. Ženíšek: Finite element solution of nonlinear elliptic problems. Numer. Math. 50 (1987) 451-475.

21. L. Gastaldi: Mixed finite element methods in fluid-structure systems. Numer. Math. 74 (1996) 153-176.

22. V. Girault and P.A. Raviart: Finite Element Methods for Navier-Stokes Equations. Springer-Verlag, 1986.

23. P. Grisvard: Elliptic Problems on Nonsmooth Domains. Pitman, Boston, 1985.

24. M. Hamdi, Y. Ousset, and G. Verchery: A displacement method for the analysis of vibrations of coupled fluid-structure systems. Internat. J. Numer. Methods Eng. 13 (1978) 139-150.

25. E. Hernández and R. Rodríguez: Finite element approximation of spectral problems with Neumann boundary conditions on curved domains. Math. Comp. 72 (2003) 10991115 .

26. T. Kato: Perturbation Theory for Linear Operators. Springer, Berlin, 1976.

27. L. Kiefling and G.C. Feng: Fluid-structure finite element vibrational analysis. AIAA J. 14 (1976) 199-203.

28. M.P. Lebaud: Error estimate in an isoparametric finite element eigenvalue problem. Math. Comp. 63 (1994) 17-40.

29. H.J-P. Morand and R. Ohayon: Fluid-structure Interactions. John Wiley \& Sons, New York, 1995.

30. R. Ohayon and C. Soize: Structural Acoustics and Vibration. Academic Press, New York, 1998.

31. R. Rodríguez and J. Solomin: The order of convergence of eigenfrequencies in finite element approximations of fluid-structure interaction problems. Math. Comp. 65 (1996) 1463-1475.

32. P.A. Raviart and J.M. Thomas: Introduction à l'Analyse Numérique des Equations aux Dérivées Partielles. Masson, Paris, 1983.

33. P.A. Raviart and J.M. Thomas: A mixed finite element method for second order elliptic problems. In: Mathematical Aspects of Finite Element Methods. Lecture Notes in Mathematics 606. Springer Verlag, Berlin, Heidelberg, New York, 1977, pp. 292-315.

34. G. Strang and G.J. Fix: An Analysis of the Finite Element Method. Prentice-Hall, Englewood Cliffs, NJ, 1973.

35. M. Vanmaele and A. Ženíšek: External finite element approximations of eigenvalue problems. Math. Model. Numer. Anal. (M²AN) 27 (1993) 565-589.

36. M. Vanmaele and A. Ženíšek: External finite-element approximations of eigenfunctions in the case of multiple eigenvalues. J. Comput. Appl. Math. 50 (1994) 51-66.

37. M. Vanmaele and A. Ženíšek: The combined effect of numerical integration and approximation of the boundary in the finite element methods for the eigenvalue problems. Numer. Math. 71 (1995) 253-273.

38. X. Wang and K.J. Bathe: Displacement/pressure based mixed finite element formulation for acoustic fluid-structure interaction problems. Internat. J. Numer. Methods Eng. 40 (1997) 2001-2017.

39. A. Ženíšek: Nonlinear Elliptic and Evolution Problems and their Finite Element Approximation. Academic Press, London, 1990. 